\title{
Extensional and torsional pipe flow
}

\author{
Francisco Marques $\odot$ and Alvaro Meseguer \\ Departament de Física, Universitat Politècnica de Catalunya, Barcelona 08034, Spain
}

(Received 26 April 2019; published 31 October 2019)

\begin{abstract}
We study the dynamics of a viscous fluid confined within an elastic pipe that is being stretched and twisted in the axial and azimuthal directions, respectively. A comprehensive numerical exploration of self-similar flows has been conducted for a wide range of stretching and twisting acceleration rates. Up to eight different families of steady flows have been identified and tracked. For purely axial stretching, the presented computations recover the results obtained by former authors in the past. However, the twisting mechanism, seen as a homotopy parameter, has been crucial in this study to explain how different families of equilibria interact and azimuthal swirl is induced. The explorations conclude that stable time-periodic flows may also take over the dynamics when the pipe is contracting in the axial direction, with mild or absent azimuthal twist. These time-periodic flows may destabilize through different codimension-two bifurcation scenarios, sometimes leading to unstable tori that have been genuinely identified by means of edge-tracking techniques.
\end{abstract}

DOI: 10.1103/PhysRevFluids.4.103908

\section{INTRODUCTION}

Stretching-shrinking pipes or channels appear in the mathematical modeling of physiological fluid flows [1,2]. These models usually assume self-similarity of the Navier-Stokes solution in the streamwise or spanwise direction in which the wall is being deformed. For a comprehensive survey of self-similar and other exact Navier-Stokes flows, we refer the reader to the monograph by Drazin and Riley [3]. The dynamics of incompressible fluids bounded by axially stretching pipes was first studied in [4]. Other authors have also considered the combined effect of stretching and porous pipe walls, allowing for fluid suction [5,6]. These works have addressed the possibility of generating azimuthal swirl due to the aforementioned mechanisms of stretching and suction injection. Most of these classical works in self-similar flows focused on the study of steady solutions. However, the origin of flow-induced oscillations in elastic-walled biological conduits has recently attracted much attention [7,8].

In the present work, we will study the behavior of the fluid when the pipe is being axially stretched and, simultaneously, azimuthally twisted. In our exploration, the azimuthal torsion plays the role of a homotopy factor that allows one to unfold the parameter space and its solutions. This unfolding paves the way to answer many open questions regarding the apparent disconnection or absence of solution branches formerly reported by some authors in the past for purely axially stretching pipes [4]. Our exploration therefore requires tracking families of self-similar flows when the stretching and torsional effects are varied independently. For this purpose, we have developed a wide variety of numerical techniques, such as time integrators of the governing equations or Newton-Krylov-Poincaré continuation schemes, that allow tracking of the steady or time-periodic flows, regardless of their stability. In the parameter space, these flows may undergo instabilities leading to the emergence of new flow regimes. To forecast these bifurcations, it has also been necessary to implement Arnoldi-Poincaré linear stability analyses for the computation of eigenvalues or Floquet exponents of the aforementioned equilibrium flows or periodic orbits, respectively. This approach, based on a dynamical systems perspective, has been proven very 

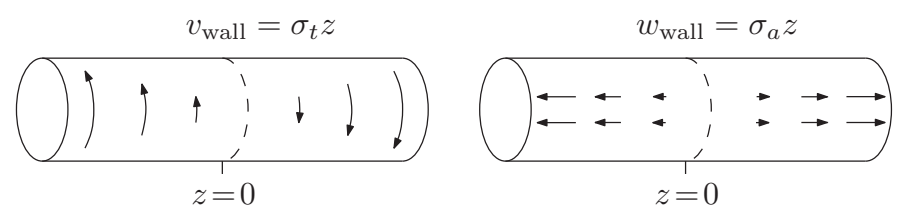

FIG. 1. Schematics and notation of the extensional pipe, in nondimensional variables.

successful in the understanding of complex dynamics arising in self-similar flows within plane geometries [9-11].

The paper is structured as follows. In Sec. II, we formulate the self-similar Navier-Stokes problem and briefly describe the symmetries of its solutions as well as the numerical techniques used to their discretization. Further details regarding formulation, symmetries, and numerics can be found in Appendix A. In Sec. III, we describe the different families of steady flows for small stretching and twisting rates. That section also devotes part of the exploration to recover the twist-free flows formerly computed in the past by Brady and Acrivos. In Sec. IV, we carry out a comprehensive exploration of the cusp bifurcations that lead to the merging of different solution branches. In this section, we also address the mathematical singularities that some of these branches may exhibit for some particular stretching-twisting rates. Section V is strictly devoted to the instability bifurcation mechanism that may lead to time-periodic flows when the pipe is mainly shrinking with very small or nearly absent twist. This section describes in detail what kind of periodic orbits may emerge, their range of existence, and their stability. Finally, Sec. VI explores the presence of quasiperiodic flows originated through Neimark-Sacker bifurcations exhibited by some of the periodic orbits previously studied in Sec. V.

\section{SELF-SIMILAR FORMULATION}

Let us consider an infinitely long cylindrical pipe with elastic wall of radius $\ell$, filled with an incompressible fluid of kinematic viscosity $v$. In our problem, the pipe wall is simultaneously being stretched in the axial direction and twisted azimuthally. The axial $w^{*}$ and azimuthal $v^{*}$ velocities of the pipe wall are linearly proportional to the axial coordinate $z^{*}$, that is, $w^{*}=z^{*} E_{a}$ and $v^{*}=z^{*} E_{t}$, where $E_{a}$ and $E_{t}$ are the axial stretching and azimuthal torsion rate factors, respectively. All variables are rendered nondimensional using $\ell$ and $\ell^{2} / v$ as units of length and time, respectively. To measure the strength of the total stretching and twisting of the pipe wall, we introduce the Reynolds number,

$$
R=\frac{\ell^{2}}{v} \sqrt{E_{a}^{2}+E_{t}^{2}}=\sqrt{\sigma_{a}^{2}+\sigma_{t}^{2}}
$$

where $\sigma_{a}=\ell^{2} E_{a} / v$ and $\sigma_{t}=\ell^{2} E_{t} / v$ are the nondimensional stretching and torsional rates, respectively. Figure 1 depicts the resulting azimuthal and axial speed profile distributions of the pipe wall in the nondimensionalized variables. Following [11], we also introduce the parametrization $\sigma_{a}=R \cos \alpha$ and $\sigma_{t}=R \sin \alpha$, with $\alpha \in[0,2 \pi]$, where $\tan \alpha=\sigma_{t} / \sigma_{a}$ measures the ratio between the wall stretching and torsion rates.

In what follows, we formulate the problem in cylindrical coordinates $(r, \theta, z)$, with $(u, v, w)$ the velocity field components in the radial, azimuthal, and axial directions. Regularity of the flow field at the axis requires $u$ and $v$ to be of the form $u=r G_{1}\left(r^{2}\right)$ and $v=r G_{2}\left(r^{2}\right)$, whereas $w$ must be of the form $w=G_{3}\left(r^{2}\right)$; see [12-14]. Following [6,15], we make use of the auxiliary variable $x=2 r^{2}-1$, mapping the unit radial interval $r \in[0,1]$ to the canonical domain $x \in[-1,+1]$ where we expand the vector fields in terms of orthogonal polynomials.

The self-similar ansatz assumes axisymmetric flow, with axial and azimuthal velocity components proportional to the axial coordinate $z$, as suggested by the boundary conditions. The 
aforementioned ansatz, along with the incompressibility condition, leads to a velocity field of the form

$$
\begin{gathered}
u(r, \theta, z, t)=r F(x, t), \quad v(r, \theta, z, t)=r z H(x, t), \\
w(r, \theta, z, t)=-\frac{z}{r} \partial_{r}\left(r^{2} F\right)=-2 z \partial_{x}[(x+1) F(x, t)],
\end{gathered}
$$

where $H$ is the angular velocity $\omega=v / r$ of a fluid element at $z=1$, i. e., $H=\left.\omega\right|_{z=1}$. Substituting the ansatz (2) into the Navier-Stokes equations and eliminating the pressure field from the formulation results in the governing equations for $F$ and $H$ :

$$
\begin{aligned}
D D_{+} F_{t}+2\left|\begin{array}{rr}
(x+1) F & D_{+} F \\
D D_{+} F & D^{2} D_{+} F
\end{array}\right|-\frac{1}{4} H^{2} & =8\left(D D_{+}\right)^{2} F, \\
H_{t}+2\left|\begin{array}{ll}
F & D_{+} F \\
H & D_{+} H
\end{array}\right| & =8 D D_{+} H, \\
F(1, t) & =0, \quad D F(1, t)=-\frac{1}{4} \sigma_{a}, \quad H(1, t)=\sigma_{t},
\end{aligned}
$$

where the $r$ derivatives have been replaced in terms of the $x$ derivatives, using $D=\partial_{x}$ and $D_{+}=$ $D(x+1)$; see Appendix A for details.

The stream function and pressure can be expressed in terms of the $F$ and $H$ variables:

$$
\begin{gathered}
\psi(x, z, t)=-\frac{1}{2} z(x+1) F(x, t), \\
p(r, z, t)=z^{2} p_{2}(x, t)+p_{0}(x, t), \\
p_{2}=D_{+} F_{t}+2(x+1) F D D_{+} F-2\left(D_{+} F\right)^{2}-8 D_{+} D D_{+} F, \\
p_{0}=-\frac{1}{4} \int\left(F_{t}+2 F D_{+} F-F^{2}-8 D D_{+} F\right) d x .
\end{gathered}
$$

Since $D p_{2}=H^{2} / 4$, there exists a constant $\beta$ given by

$$
\beta=p_{2}(x, t)-\frac{1}{4} \int_{-1}^{x} H^{2} d x,
$$

which has been used in previous studies of this problem [4]. As a measure of the azimuthal swirl of the flow, we will use the azimuthal mass flux, given by

$$
\phi_{\theta}=\int_{0}^{1} v(r) d r=\frac{1}{2} \sigma_{t}+\frac{1}{4} \int_{-1}^{1} h(x) d x .
$$

\section{A. Symmetries}

The pipe geometry is invariant under rotations around the pipe axis, reflections about planes orthogonal to the pipe ( $z$ constant), and reflections about meridional planes ( $\theta$ constant). The selfsimilar solutions are invariant under rotations around the pipe axis and the reflection about $z=0$; therefore, the only nontrivial symmetry that remains is the reflection $K_{\theta}$ about the meridional plane $\theta=0$, acting on the polar coordinates as $K_{\theta}(r, \theta, z)=(r,-\theta, z)$. The corresponding action on the velocity field, angular velocity, stream function, pressure, and self-similar variables $F$ and $H$ are

$$
K_{\theta}(u, v, w, \omega, \psi, p, F, H)(r, z)=(u,-v, w,-\omega, \psi, p, F,-H)(r, z) .
$$

The boundary conditions (3c) are $K_{\theta}$ equivariant only for $\sigma_{t}=0$, i.e., in the absence of twisting on the pipe wall, corresponding to $\alpha=0, \pi . K_{\theta}$ transforms a solution for a given value of $\alpha$ into 
another solution for a different value of $\alpha$ : $-\alpha$, or what is the same, $2 \pi-\alpha$. The action of $K_{\theta}$ on the parameters and constants of the problem is

$$
K_{\theta}\left(\sigma_{a}, \sigma_{t}, R, \alpha, \beta, \phi_{0}\right)=\left(\sigma_{a},-\sigma_{t}, R,-\alpha, \beta,-\phi_{0}\right) .
$$

\section{B. Numerical techniques}

In order to discretize the sought fields $F$ and $H$ in (3), we perform the splitting

$$
F(x, t)=f(x, t)+f_{b}, H(x, t)=h(x, t)+h_{b},
$$

where $f_{b}=\frac{1}{4} \sigma_{a}(1-x)$ and $h_{b}=\sigma_{t}$ are two basic profiles satisfying the stretching and torsional boundary conditions at the wall, whereas $f(x, t)$ and $h(x, t)$ are two sought-after fields satisfying homogeneous boundary conditions. Introducing (12) in (3) leads to the governing equations for $f$ and $h$ :

$$
\begin{gathered}
D D_{+} f_{t}+N_{f}(f, h)=8\left(D D_{+}\right)^{2} f, \quad h_{t}+N_{h}(f, h)=8 D D_{+} h, \\
f(1, t)=D f(1, t)=h(1, t)=0,
\end{gathered}
$$

where the nonlinear terms are

$$
N_{f}=2\left|\begin{array}{cc}
(x+1)\left(f+f_{b}\right) & D_{+}\left(f+f_{b}\right) \\
D D_{+} f-\sigma_{a} / 2 & D^{2} D_{+} f
\end{array}\right|-\frac{1}{4}\left(h+\sigma_{t}\right)^{2}, \quad N_{h}=2\left|\begin{array}{cc}
f+f_{b} & D_{+}\left(f+f_{b}\right) \\
h+\sigma_{t} & D_{+} h+\sigma_{t}
\end{array}\right| .
$$

We approximate $f$ and $h$ via spectral expansions based on modulated Legendre polynomials satisfying suitable homogeneous boundary conditions,

$$
\begin{aligned}
f(x, t) & =\sum_{m=0}^{M-1} a_{m}(t) \Phi_{m}(x), \quad \Phi_{m}(x)=(1-x)^{2} P_{m}(x), \quad \Phi_{m}(1)=\Phi_{m}^{\prime}(1)=0, \\
h(x, t) & =\sum_{m=0}^{M-1} c_{m}(t) \Psi_{m}(x), \quad \Psi_{m}(x)=(1-x) P_{m}(x), \quad \Psi_{m}(1)=0,
\end{aligned}
$$

where $P_{m}(x)$ is the $m$ th Legendre polynomial. The expansions above are formally introduced in the governing equations (13) and then projected on the corresponding basis (Galerkin method) to obtain a system of nonlinear ordinary differential equations for the expansion coefficients $a_{m}$ and $c_{m}$ :

$$
\begin{aligned}
\sum_{m=0}^{M-1} A_{m n} \dot{a}_{n}+N_{1, m}\left(a, c, \sigma_{a}, \sigma_{t}\right) & =\sum_{m=0}^{M-1} L_{1, m n} a_{n}, \\
\sum_{m=0}^{M-1} G_{2, m n} \dot{c}_{n}+N_{2, m}\left(a, c, \sigma_{a}, \sigma_{t}\right) & =\sum_{m=0}^{M-1} L_{2, m n} c_{n},
\end{aligned}
$$

for $m=1,2, \ldots, M-1$. The explicit form of the matrices $A, G_{2}, L_{1}$, and $L_{2}$, along with the vectors $N_{1}$ and $N_{2}$, is given in Appendix A. The number $M$ of Legendre polynomials used in the expansions strongly depends on the particulars of the different solutions and the values of $(R, \alpha)$. In the present work, $M \in[70,200]$; the criteria used to choose a suitable value of $M$ is given in Appendix B.

The time integration of (17) is based on an implicit-explicit second order method: implicit second-order backward differentiation formula scheme for the linear part, and explicit second order for the nonlinear terms. The steady solutions have been obtained using the Newton method and a pseudo-arclength continuation scheme in parameter space $(R, \alpha)$. Periodic solutions have been computed by implementing a pseudo-arclength Newton-Krylov-Poincaré continuation method $[11,16,17]$. Linear stability analyses of fixed points and periodic orbits have been carried out using 


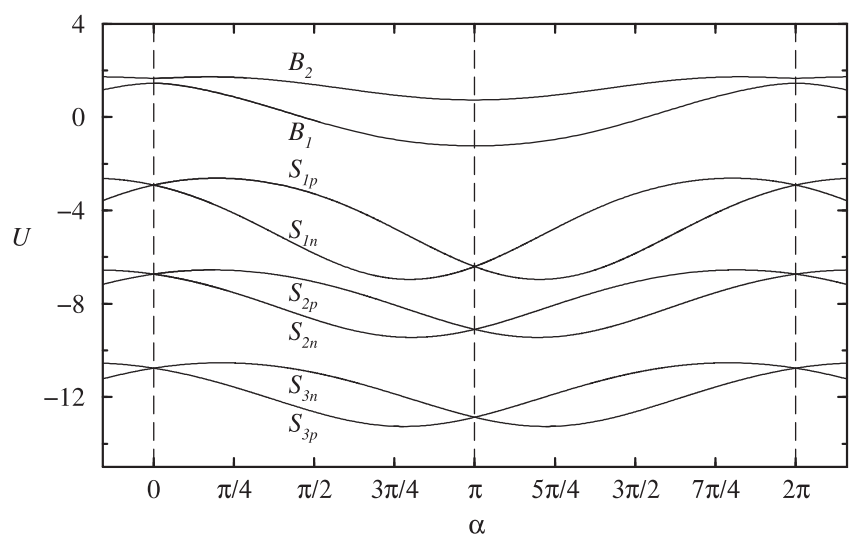

FIG. 2. Families of steady solutions at $R=10$.

Krylov-Arnoldi methods [18] tailored for the linearized Poincaré maps. A detailed description of these methods can be found in [11].

\section{FAMILIES OF STEADY SOLUTIONS}

Brady and Acrivos [4] found six different families of solutions at small $R$, for $\alpha=0$. Two of the solutions have zero azimuthal velocity, $v=0$, and the other solutions have swirl, in spite of having $\alpha=0$, and no torsion applied on the cylinder wall. We have continued these solutions for all values of $\alpha$ and the result is shown in Fig. 2, for $R=10$. We have also found two families of solutions with swirl that exist at small $R$ for all $\alpha$ values, called $S_{3 p}$ and $S_{3 n}$; these solutions have been found using continuation in $R$ and $\alpha$ because all branches of solutions become connected when increasing $R$, via fold bifurcations that will be described in Sec. IV. In order to characterize the different solutions, the values of the velocity components in cylindrical coordinates at $\left(r_{0}, z_{0}\right)=(1 / \sqrt{2}, 1), U, V$, and $W$ will be used. The radius $r_{0}$ divides the pipe section in two annular regions of equal area. We will also use $W_{0}$ and $\Omega_{0}$, the axial velocity and the angular velocity at the pipe center, and the pressure parameter $\beta$ (8).

The eight solutions exist for all $\alpha$ values, and there are no connections between the different branches at this low- $R$ value. The solutions without swirl for $\alpha=0$ are called $B_{1}$ and $B_{2} ; B_{1}$ is a stable solution that smoothly becomes the zero solution $u=v=w=0$ of the Navier-Stokes equations at $R=0$. The other solutions are unstable. The solutions with swirl will be called $S_{1}, S_{2}$, and $S_{3}$, and they exist in two forms, with positive or negative values of $v, S_{i p}$, and $S_{i n}$ for $i=1-3$. The symmetry operation $K_{\theta}$ transforms one into the other: $K_{\theta} S_{i p}(\alpha)=S_{i n}(2 \pi-\alpha)$. The solutions without swirl transform into the same family: $K_{\theta} B_{i}(\alpha)=B_{i}(2 \pi-\alpha)$. These symmetry properties are clearly seen in Fig. 2. There are two lines of symmetry in the figure, indicated by dotted lines, corresponding to $\alpha=0$ and $\pi$. The $B_{i}$ solutions at these particular values are $K_{\theta}$ symmetric.

For a quantitative comparison with previous works and as a check of the numerical methods used, we have computed the eight solutions for $\alpha=0$, the purely stretching case, up to $R=3000$; there are four additional solutions without swirl appearing when increasing $R$, labeled $B_{i}, i=3-6$, that were also reported in [4]. They are shown in Fig. 3(a), which plots $U$ as a function of $R$. Figure 3(b) is a reconstruction of Fig. 13 in [4], depicting in logarithmic scale the pressure parameter $2 \beta / R$ versus $R$; the agreement is very good. The branches $B_{3}$ and $B_{4}$, which appear for $R \geqslant 147$, will be discussed later. There are also two additional branches without swirl, $B_{5}$ and $B_{6}$, that appear for $R \geqslant 730$, which are unstable (with two and three positive eigenvalues, respectively). In the parameter range $(\alpha, R)$ that we have explored, these two branches do not exist or, when they do, they do not interact with the other branches and do not display any new bifurcation apart from the 
(a)

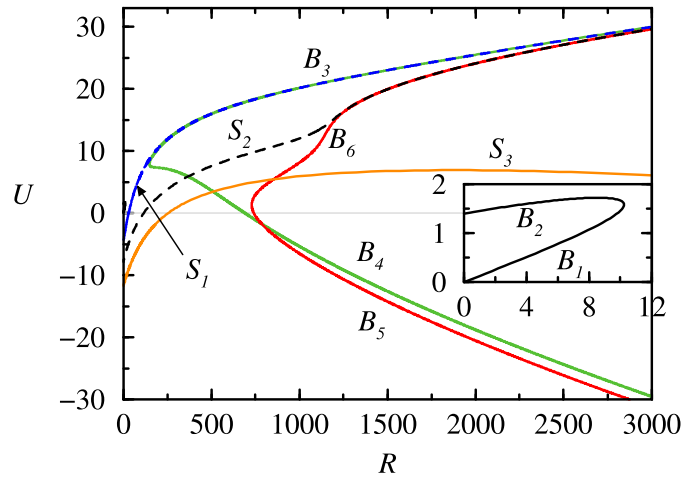

(b)

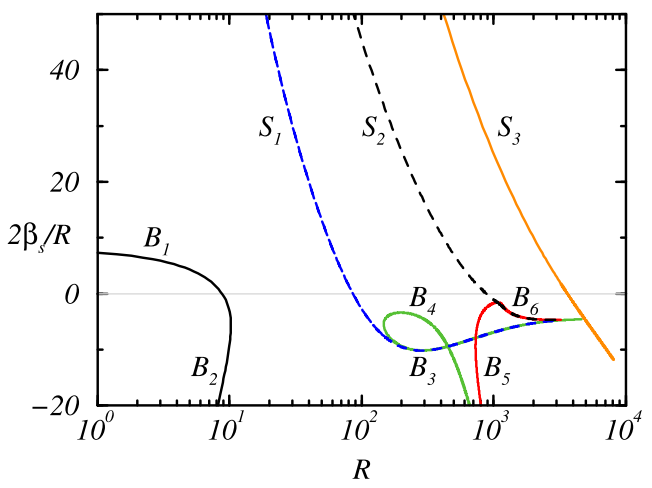

FIG. 3. Branches of steady solutions at $\alpha=0$, following Brady and Acrivos [4].

saddle node between $B_{5}$ and $B_{6}$ shown in Fig. 3 at about $R=729.26$. Therefore, they will not be considered in the rest of this study.

The flow structure of the $B_{1}$ and $B_{2}$ families is illustrated in Fig. 4, where five equispaced solutions in $\alpha \in[0, \pi]$ are shown; the solutions for $\alpha \in[\pi, 2 \pi]$ are the same due to the $K_{\theta}$ symmetry: $K_{\theta} B_{i}(\alpha)=B_{i}(2 \pi-\alpha)$. The panels show radial profiles of the radial $u$, axial $w$, and angular $\omega=v / r$ velocities. They also show a tridimensional view of $w$ at the $z=1$ circular section of the pipe, where the color indicates the $\omega$ values. The solutions $B_{1}$ are almost in solid body rotation $(\omega=$ constant $)$, and the axial velocity in the interior of the pipe is of the same order as the applied stretching $\sigma_{a}=R \cos \alpha$, but of opposite sign because the axial net mass flux in the pipe is zero. This is due to the self-similar form of the solution (2), which forces $w=0$ at $z=0$ and, due to the incompressibility, the mass flux in any other section is also zero. Therefore, for $B_{1}$ solutions, the flow in the interior responds passively to the applied stretching and twisting of the wall, keeping $\omega(r) \approx \sigma_{t}=R \sin \alpha$, and the axial velocity around the axis changes sign in order to satisfy incompressibility. This is more clearly seen in the accompanying movie, movie1.mp4 (see Supplemental Material [24]), showing the variation of the $w$ and $\omega$ profiles on the pipe section $z=1$ as a function of $\alpha \in[0,2 \pi]$.

The structure of $B_{2}$ is different. Regardless of the value of $\alpha$, all the solutions in this family have a jet at the axis, moving towards $z=0$ with a speed substantially larger than $R$. This axial jet is in solid body rotation, with an angular speed remarkably smaller than $R$. When changing $\alpha$, all variations in $\omega$ and $w$ are concentrated within a boundary layer near the wall. The wall's stretching and twisting have a small effect within the pipe's core, changing only the intensity of the axial jet, but not its orientation. The maximum strength of the jet takes place precisely at $\alpha=\pi$ for pure axial shrinking of the pipe wall. See movie2.mp4 in the Supplemental Material [24].

The flow structure of the $S_{1}$ family of solutions is illustrated in Fig. 5, describing the $S_{1 p}$ branch; the $S_{1 n}$ branch can be obtained applying the symmetry $K_{\theta} S_{i n}(\alpha)=S_{i p}(2 \pi-\alpha)$, which changes the sign of $\omega$ while keeping the sign of $u$ and $w$. Regardless of the value of $\alpha$, all the solutions in this family have a jet at the axis, moving away from $z=0$ with a speed substantially larger than $R$. This axial jet is in solid body rotation, with an angular speed substantially larger than $R$, and twice as large as $w$. The wall's stretching and twisting have a moderate effect within the pipe's core, changing only the intensity of the axial jet, but not its orientation and twist. The maximum strength of the jet takes place at about $\alpha=5 \pi / 4$ for axial shrinking and twisting of the pipe wall of equal intensity. The minimum jet strength takes place at about $\alpha=\pi / 4$ for axial stretching and twisting of the pipe wall of equal intensity.

The flow structure of the $S_{2}$ family of solutions is illustrated in Fig. 6, describing the $S_{2 p}$ branch; the $S_{2 n}$ branch can be obtained by applying the symmetry $K_{\theta}$. Regardless of the value of $\alpha$, all 

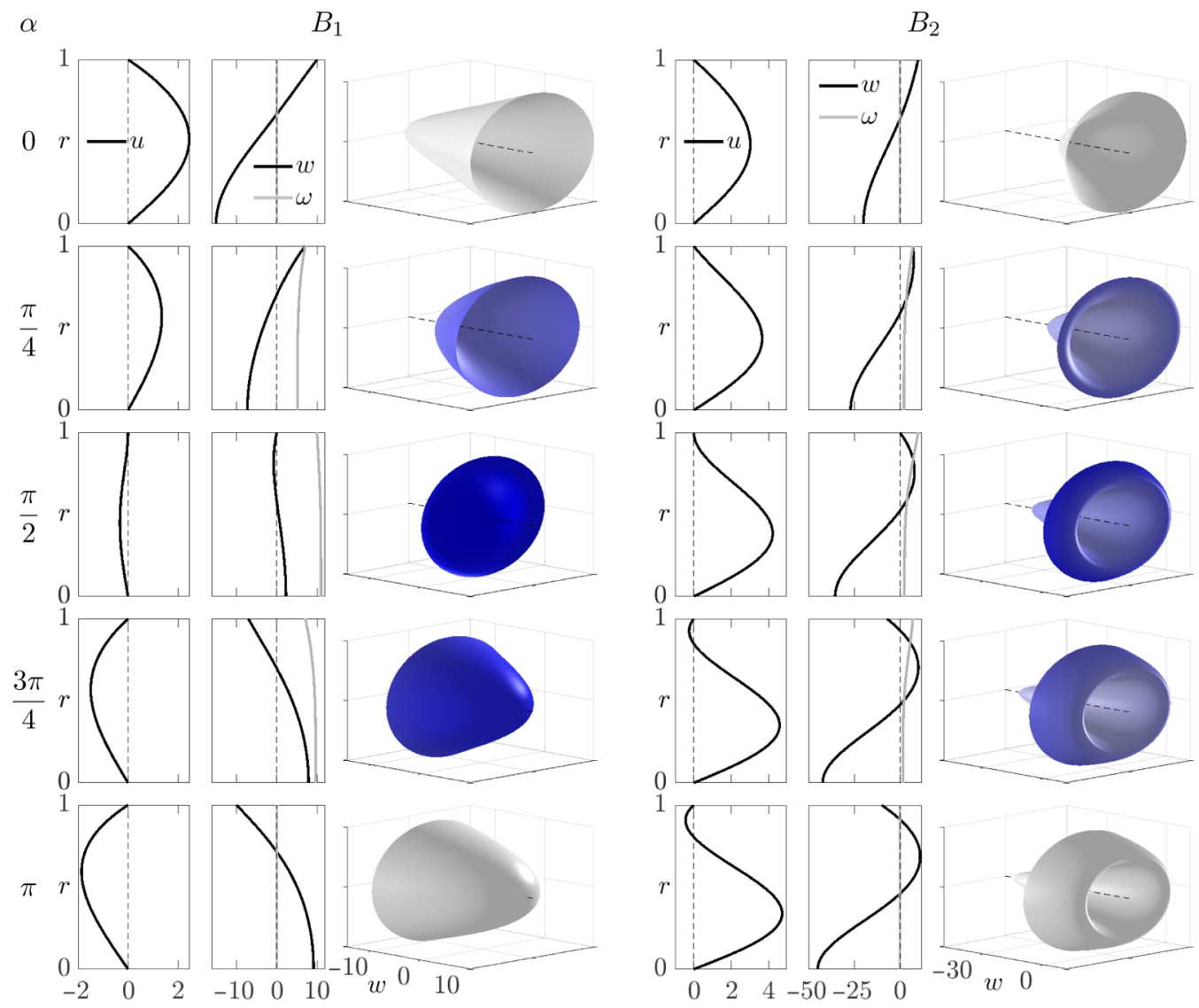

FIG. 4. Structure of the steady solutions $B_{1}$ and $B_{2}$ at $R=10$, for the $\alpha$ values indicated. The first two panels show radial profiles of $u, w$, and $\omega$ at $z=1$. The third panel shows a tridimensional view of $w$ at the $z=1$ circular section of the pipe; the dashed horizontal line is the pipe axis $(r=0)$. The color indicates the $\omega$ values: blue for $\omega>0$, light gray for $\omega=0$, and yellow for $\omega<0$. See the Supplemental Material [24] for animations of $B_{1}$ and $B_{2}$, varying $\alpha$ continuously (movie1.mp4 and movie2.mp4).

the solutions in this family have a jet at the axis, moving towards $z=0$ with a speed substantially larger than $R$. This axial jet is in solid body rotation, with a moderate angular speed comparable to $R$. The maximum value of $\omega$, one order of magnitude larger than $R$, is located in a rotating inner shear layer at about $r \in[0.5,0.7]$, which also has a significant axial velocity moving away from $z=0$. The wall's stretching and twisting have a moderate effect within the pipe's core, changing only the intensity of the axial jet and the inner shear layer, but not their orientation and twist. The maximum (minimum) strength of the axial jet and the inner shear layer takes place at about $\alpha=5 \pi / 4(\alpha=\pi / 4)$; this axial jet becomes very strong when increasing $R$ in this region.

The flow structure of the $S_{3}$ family of solutions is illustrated in Fig. 7, describing the $S_{3 p}$ branch. The solutions in this family have a very intense jet at the axis, moving away $z=0$ with an axial speed four orders of magnitude larger than $R$, and much more intense than any other family of solutions. This narrow axial jet is also rotating with high angular speed, five orders of magnitude larger than $R$. The wall's stretching and twisting have a small effect within the pipe's core, changing only the intensity of the axial jet, but not their orientation and twist. The maximum (minimum) strength of the $w$ and $\omega$ at axial jet takes place at about $\alpha=3 \pi / 4(\alpha=7 \pi / 4)$. This narrow and intense jet generates an inner boundary layer that is difficult to resolve numerically; the solutions in 

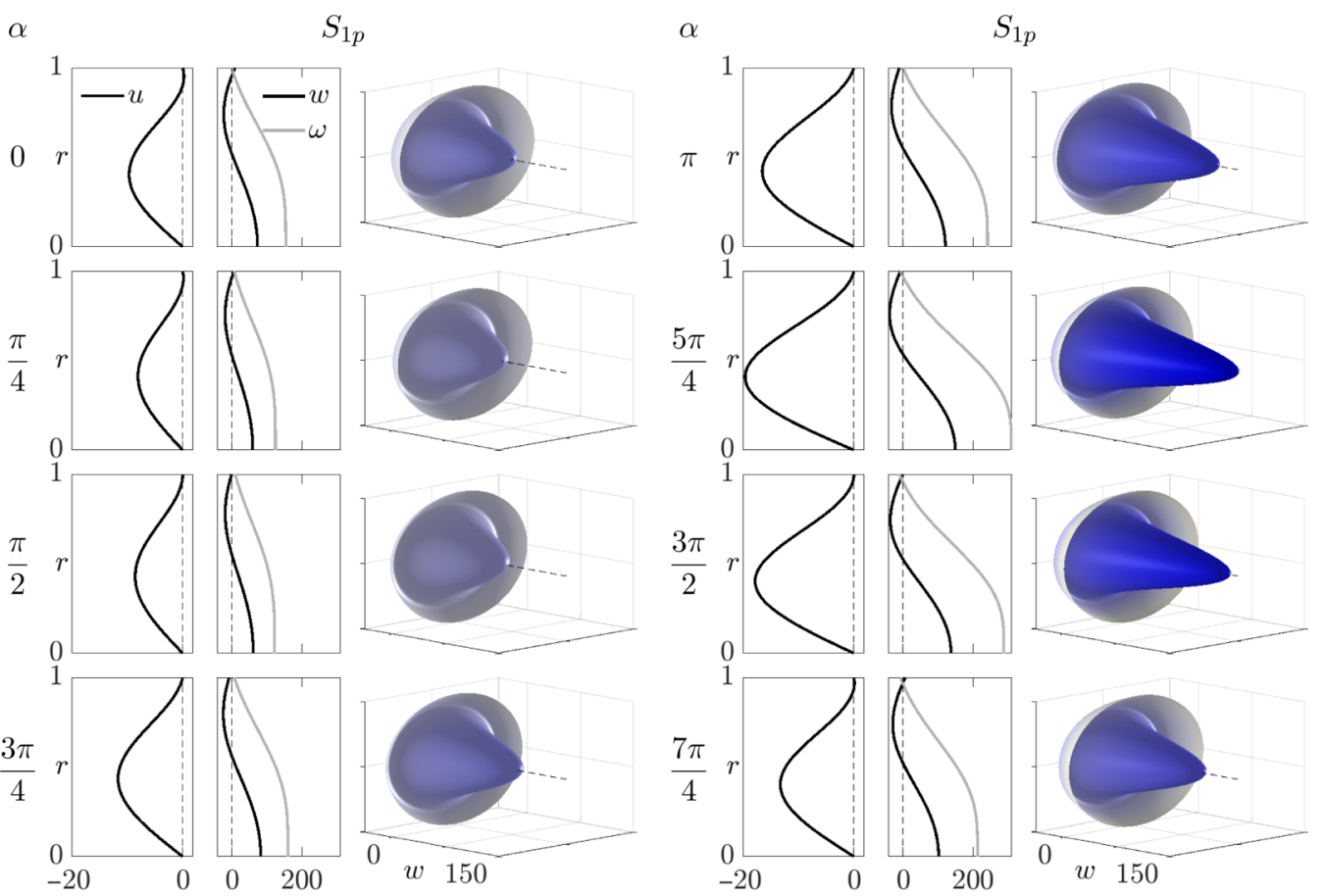

FIG. 5. Structure of the steady solutions $S_{1 p}$ at $R=10$, for the $\alpha$ values indicated. The plots follow the same conventions as in Fig. 4.
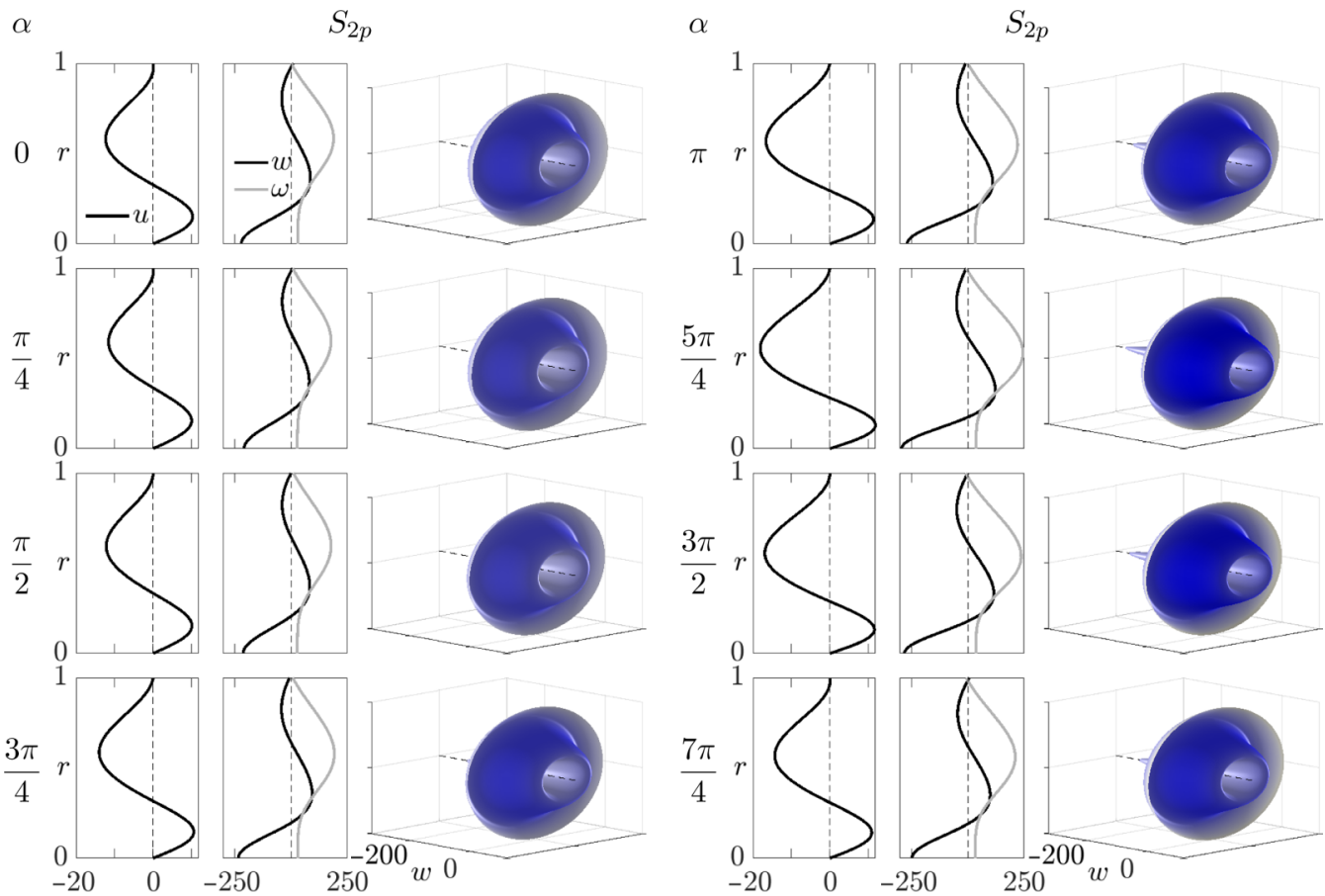

FIG. 6. Structure of the steady solutions $S_{2 p}$ at $R=10$, for the $\alpha$ values indicated. The plots follow the same conventions as in Fig. 4. 

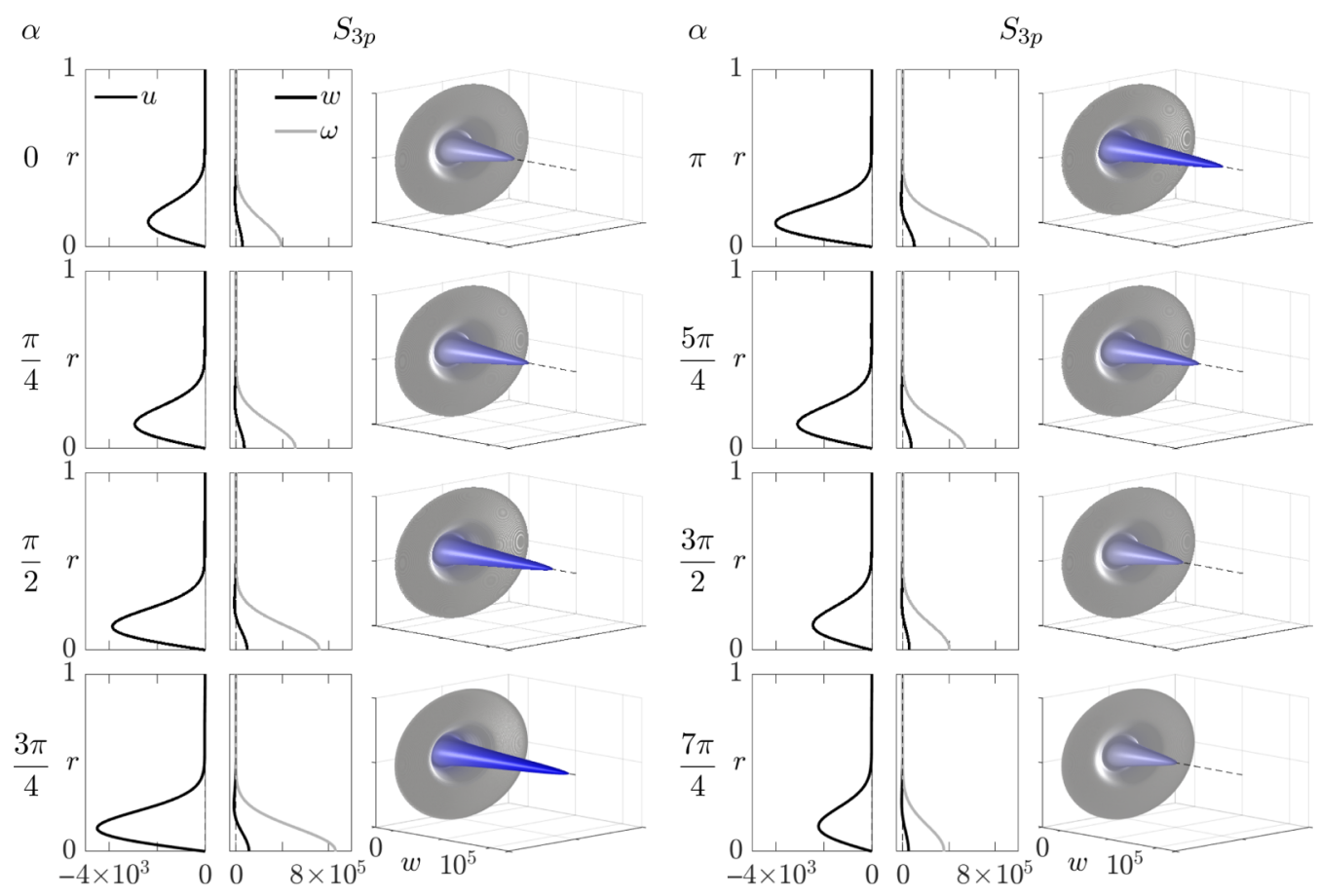

FIG. 7. Structure of the steady solutions $S_{3 p}$ at $R=10$, for the $\alpha$ values indicated. The plots follow the same conventions as in Fig. 4.

all branches have been computed using $M \in[70,200]$ Legendre polynomials in the radial direction. In some parameter ranges, it has not been possible to accurately compute solution branches $S_{2}$ and $S_{3}$, even using $M=200$ polynomials due to these intense shear layers at $r=0$. We refer the reader to Appendix B for a detailed discussion.

\section{STEADY BIFURCATIONS OF THE EIGHT BRANCHES}

When increasing the Reynolds number $R$ above $R=10$, the different branches of solutions interact: they connect via fold (also called saddle-node) bifurcations. Figure 8 shows the fold bifurcation curves we have found in the range $R \in[0,200]$. These fold curves meet in codimensiontwo cusp bifurcation points $C_{1}, C_{2}$, and the symmetric points about the lines $\alpha=0$ and $\pi$. Two branches of solutions appear or disappear at the fold bifurcation curves, and we have indicated in the figure the number of steady solutions that exist in each region, and their stability. The number of solutions is large around the lines $\alpha=0$ and $\pi$, and is smaller in between, in particular in the regions around $\alpha=3 \pi / 4$ and $5 \pi / 4$. The only stable branch is $B_{1}$, which exists only for $R$ values below the fold curves $F_{1}$. The $B_{1}$ solution becomes the zero solution at $R=0$, while the other solutions are nontrivial at $R=0$.

Figure 9 shows how the families of solutions evolve when increasing $R$, focusing on the connections between different branches and the cusp bifurcations. Comparing Fig. 2 with Fig. 9(a), we see how the branches $B_{1}$ and $B_{2}$ merge at the fold bifurcation curve $F_{1}^{L}$, and both cease to exist above it; the minimum of the $F_{1}^{L}$ is at $(\alpha, R)=(0,10.25)$. Figures $9(\mathrm{~b})$ and $9(\mathrm{c})$ show the merging of $B_{1}$ and $S_{1 p}$ at the fold bifurcation $F_{1}^{R}$, and both cease to exist above it; the minimum of the $F_{1}^{R}$ is at $(1.739,19.33)$. Figure $9(\mathrm{~d})$ shows how the intermediate branch $B_{1}$ connecting $B_{2}$ and $S_{1 p}$ ceases to exist at the cusp bifurcation $C_{1}$, for $(\alpha, R)=(0.9998,32.30)$, and the remaining branches $B_{2}$ and $S_{1 p}$ smoothly evolves one into the other. Figures 9(e) and 9(f) show the merging of $B_{2}-S_{1 p}$ 


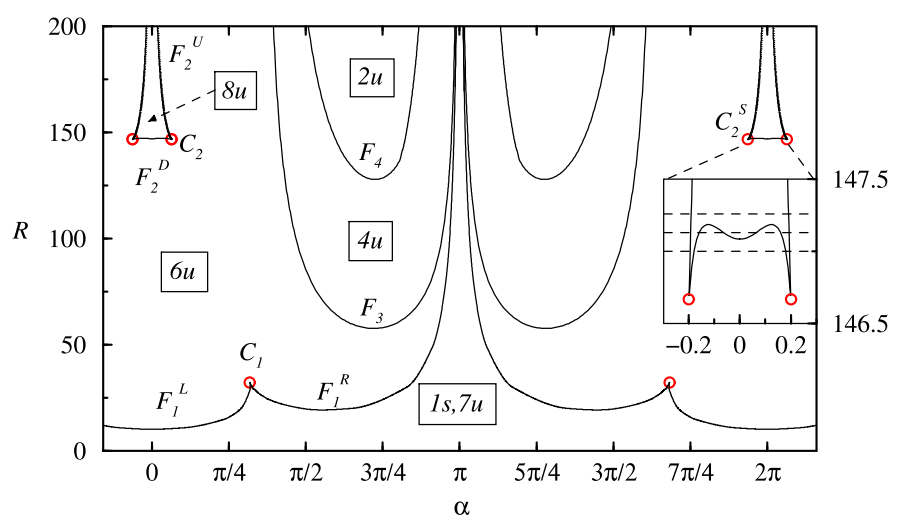

FIG. 8. Bifurcation diagram. Red circles: codimension-two cusp points. The square boxes indicate the number of steady solutions that exist in each region and their stability ( $s$ stable, $u$ unstable). The inset shows the details of the bifurcations around the $C_{2}$ cusp point.

and $S_{1 n}$ at the fold bifurcation $F_{3}$, and both cease to exist above it; the minimum of the $F_{3}$ is at (2.267, 57.81). Figures $9(\mathrm{~g})$ and $9(\mathrm{~h})$ show the merging of $S_{1 n}$ and $S_{3 n}$ at the fold bifurcation curve $F_{4}$, whose minimum is at $(2.276,127.9)$.

Some of the continuation curves in Fig. 2 for $R \geqslant 30$ do not cover all of the range $\alpha=[0,2 \pi]$, and stop at a given $\alpha$ value without any bifurcation taking place. What happens here is that some of the solutions develop narrow and intense jets at the pipe axis, and the internal boundary layer cannot be resolved satisfactorily with the numerical tool used, as discussed in detail in Appendix B. The combination of shrinking and twisting the pipe wall results in some of the solutions becoming singular: they develop a strong narrow swirling jet at the pipe axis, as shown in Appendix B, Figs. 20(a) and 20(b).

Around $\alpha=0$, i.e., the case previously studied in [4], additional branches of solutions appear when increasing the Reynolds number above $R=146.7$, as shown in Fig. 8. This solutions only exist in a narrow region around $\alpha=0$ and they disappear when moving away from the symmetry line $\alpha=0$. Therefore, the branches $B_{i}, i \geqslant 3$, are specific to the purely stretching pipe, and adding a small twisting kills these solutions; in contrast, the families $S_{i}$ with swirl persist for a wide range of $\alpha$ values. The inset in Fig. 8 shows that these solutions are born at two cusp bifurcations $C_{2}$ and $C_{2}^{S}$ (the symmetry $K_{\theta}$ transforming one into the other), happening at $(\alpha, R)=( \pm 0.19999,146.72)$.

Figure 10 shows how the $B_{3}$ and $B_{4}$ families are born and evolve when increasing $R$. Figures 10 (a) and 10 (b) illustrate how the two cusps $C_{2}$ and $C_{2}^{S}$ are formed, and the new intermediate $B_{3}$ branch appears; the new branch $B_{3}$ is delimited by two fold bifurcations, indicated with solid disks in the figure. Figure 10 (c) shows the formation of an isola at $(\alpha, R)=(0,147.1)$, with $B_{3}$ and $B_{4}$ its upper and lower branches, which meet at fold bifurcations. In Figs. 10(c) and 10(d), we observe the merging of the fold bifurcations at the ends of the isola with the lower folds associated with the cusp points, at $( \pm 0.11979,147.19)$. The new branches $B_{3}$ and $B_{4}$ join different parts of the $S_{1 n}$ and $S_{1 p}$ branches. This merging of $S_{1 n}$ and $S_{1 p}$ takes place via two fold bifurcations in the upper branch on Fig. 10(d), while $S_{1 p}, B_{4}$, and $S_{1 n}$ smoothly merge to form the lower branch.

\section{PERIODIC SOLUTIONS NEAR $\alpha=\pi$}

Time-periodic flows are frequently the result of a Hopf bifurcation emerging from a steady profile. These types of time-dependent flows play a very important role in the transition to chaotic Navier-Stokes solutions and therefore they are of utmost interest in order to predict complex dynamics. After performing systematic explorations within the plane $(\alpha, R)$, we have only identified Hopf bifurcations along the $\alpha=\pi$ line and its vicinity, that is, only in those situations when the 
(a)

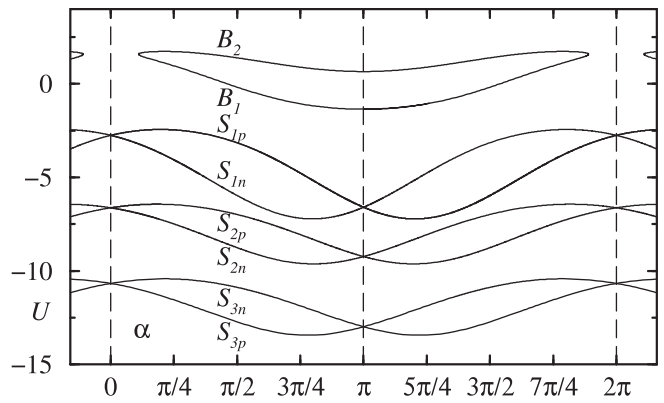

(c)

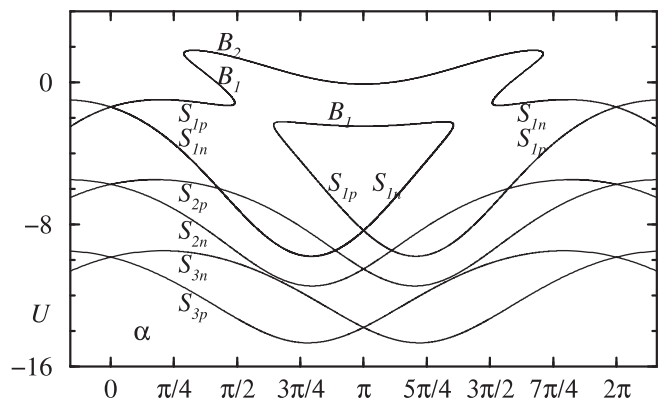

(e)

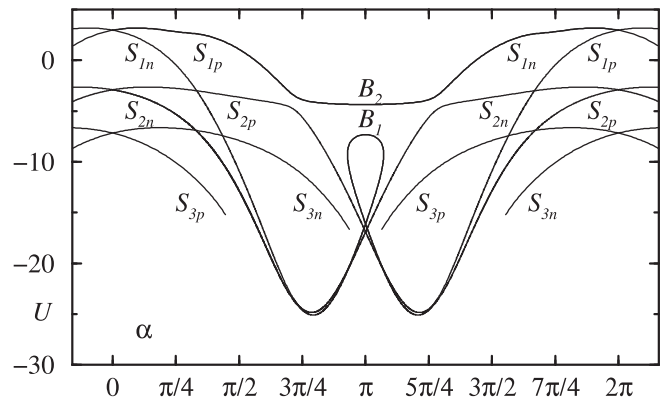

(g)

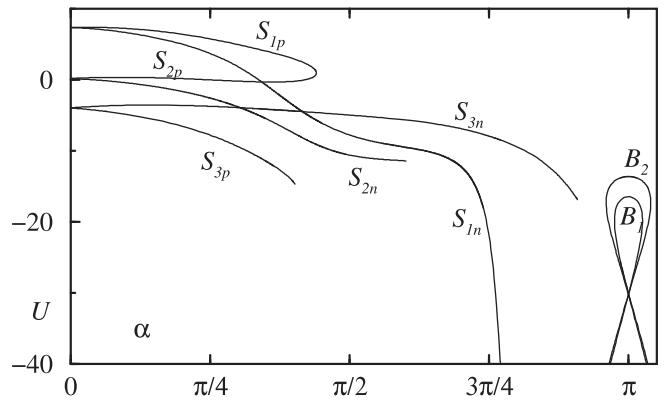

(b)

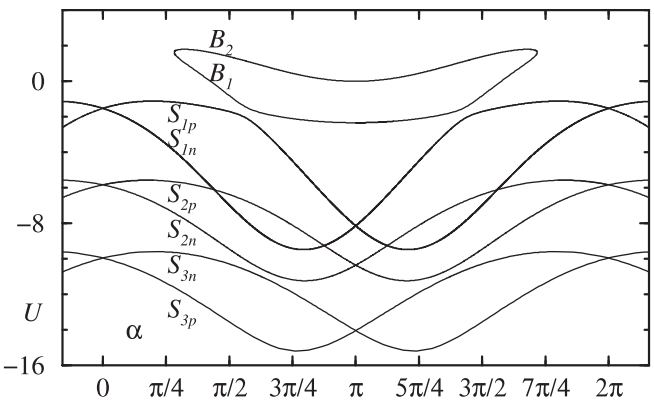

(d)

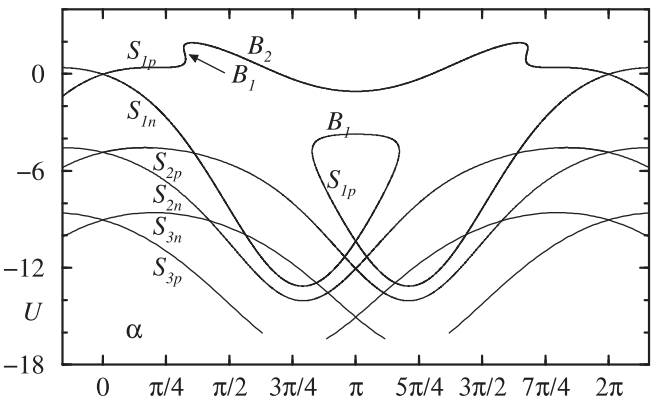

(f)

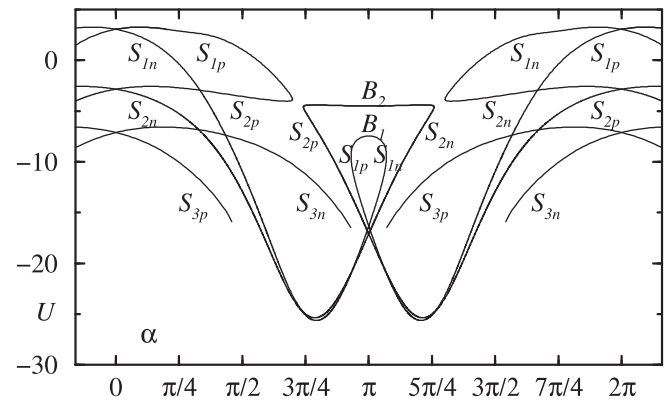

(h)

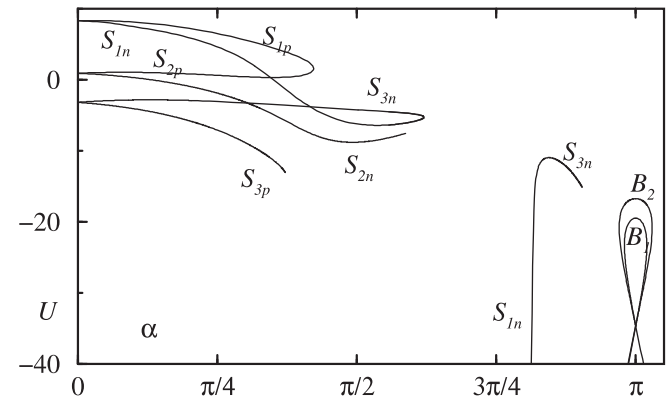

FIG. 9. Saddle-node bifurcations of the different steady branches, for $R \leqslant 200$. (a) $R=11$, (b) $R=19$, (c) $R=20$, (d) $R=30$, (e) $R=57$, (f) $R=58$, (g) $R=120$, and (h) $R=140$. 
(a)

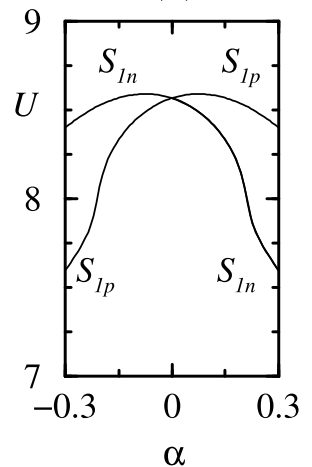

(b)

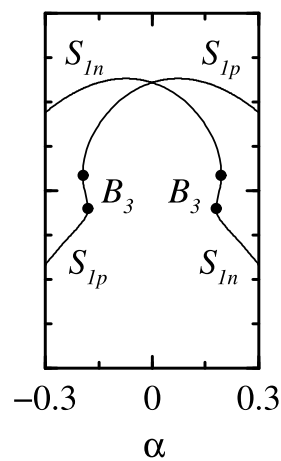

(c)

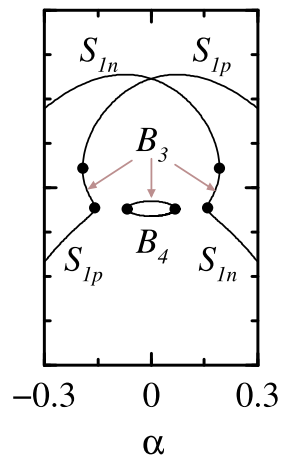

(d)

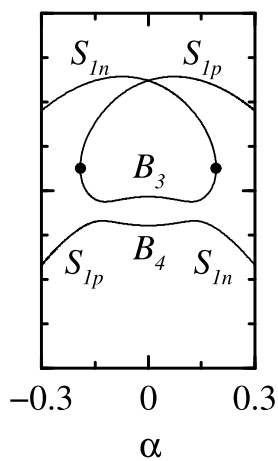

FIG. 10. Cusp bifurcations and folds around $C_{2}$. The last three panels correspond to the dashed lines in the inset of Fig. 8. The solid disks are the fold bifurcations delimiting the different branches. (a) $R=146.5$, (b) $R$ $=147$, (c) $R=147.13$, and (d) $R=147.26$.

pipe is mainly shrinking in the axial direction and in the absence of (or with small) azimuthal twist. This is consistent with the phenomena previously observed in extensional channel flows, where stable periodic orbits soon take over the dynamics when the upper and lower parallel plates shrink with the same deceleration rate. In that case, self-similar and direct numerical simulation (DNS) computations both have reported Hopf bifurcations for very low Reynolds numbers, but also a richer time-dependent dynamics when this parameter is increased, including period-doubling bifurcation scenarios leading to chaotic attractors in some cases, for example [11,19].

The branches of periodic orbits are monitored by measuring the mean azimuthal speed $V$ at $r=1 / \sqrt{2},\langle V\rangle=T^{-1} \int_{0}^{T} V(t) d t$. Figure 11(a) depicts the bifurcation diagram of $\langle V\rangle$ emerging from the basic swirl-free branch $B_{1}$ (horizontal dashed line) for $\alpha=\pi$ and $R \in$ [400,1000]. At $R=592.2$, branch $B_{1}$ exhibits a supercritical Hopf bifurcation (point $H_{1}$ ) that leads to a stable limit cycle $\Gamma$. This limit cycle still corresponds to a pure axially oscillating flow with no swirl (see movie3.mp4 in the Supplemental Material [24]). However, this periodic orbit becomes unstable at $R_{C C}=901.5$ through a pitchfork bifurcation of cycles (point $C C$ ) from which two branches [black curves $\Gamma_{p}$ and $\Gamma_{n}$ in Fig. 11(a)] of unstable limit cycles emerge. Notice that these two branches are born at a much lower Reynolds number, $R_{C F}=443.4$, at a fold bifurcation of cycles, and that they both have opposite azimuthal swirl. In other words, the pitchfork bifurcation is the result of a symmetry breaking that triggers time-periodic azimuthal swirl in the two possible orientations. At points $C F$, not only do the inner branches $\Gamma_{p}$ and $\Gamma_{n}$ emerge, but also their outer homologues $\Gamma_{\ell p}$ and $\Gamma_{\ell n}$ (also unstable), which only exist within a very narrow range of Reynolds numbers, as they rapidly reconnect with swirl equilibrium branches $S_{1 p}$ and $S_{1 n}$ (dashed curves) through Hopf bifurcations points $H_{1 p}$ and $H_{1 n}$ at $R_{H_{1}}=459.1$.

Figure 11(c) shows the time evolution of the axial $W_{0}$ and angular $\Omega_{0}$ velocities for the periodic solutions $\Gamma$ and $\Gamma_{p}$ at $R=700$ and $\alpha=\pi$, over two periods. For comparison purposes, time has been scaled with the period of the solutions, whose values are $T_{\Gamma}=5.626 \times 10^{-3}$ and $T_{\Gamma_{p}}=8.089 \times 10^{-3}$. The $\Gamma_{n}$ solution is identical with $\Gamma_{p}$ except for a change of sign in $\Omega_{0}$, due to the symmetry $K_{\theta}$ transforming $\Gamma_{p}$ into $\Gamma_{n}$; notice that $\Omega_{0}=0$ for $\Gamma$ because it is a $K_{\theta}$-symmetric solution. These periodic solutions exhibit a pulsing of the swirling axial jet; see movie3.mp4 and movie4.mp4 in the Supplemental Material [24].

Figure 11(b) shows the bifurcation diagram of the three Hopf bifurcations $H_{1}, H_{1 p}$, and $H_{1 n}$ around $\alpha=\pi$. The three Hopf curves reconnect at codimension-two fold-Hopf $F H$ and $F H^{*}$ points, where the Hopf curves meet the fold curves $C F$ and $C F^{*}$. The Hopf bifurcations $H_{1 p}$ and $H_{1 n}$, subcritical at $\alpha=\pi$, become supercritical at two Bautin codimension-two points $B a$ and $B a^{*}$, when 
(a)

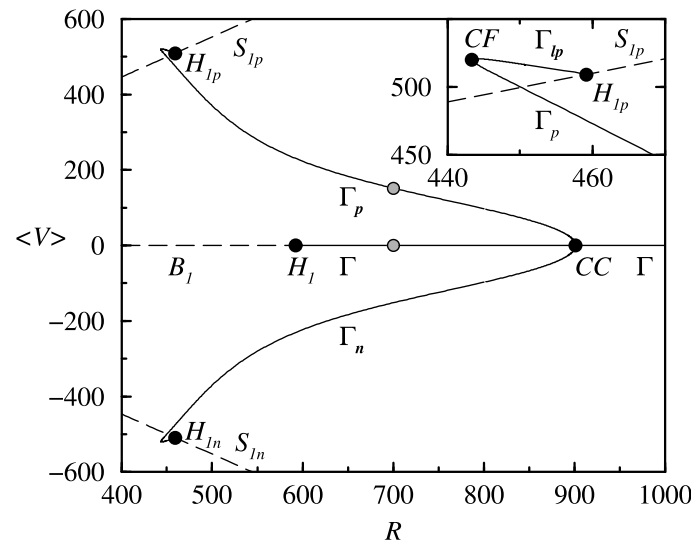

(c) $\Gamma, \Gamma_{p}$ at $(\alpha, R)=(\pi, 700)$

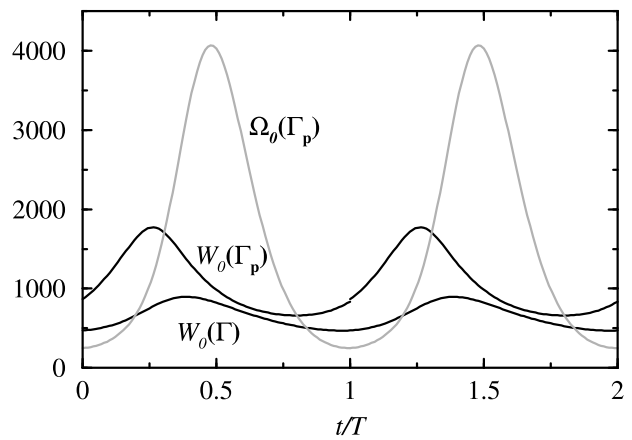

(b)

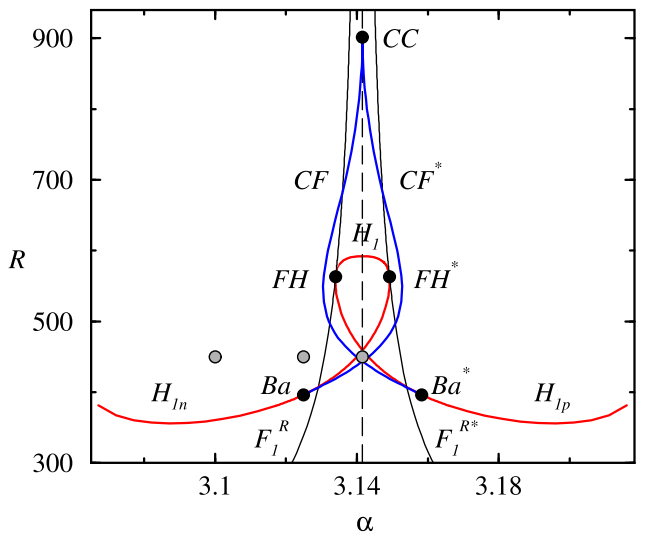

(d) $\Gamma_{n}$ at $R=450$

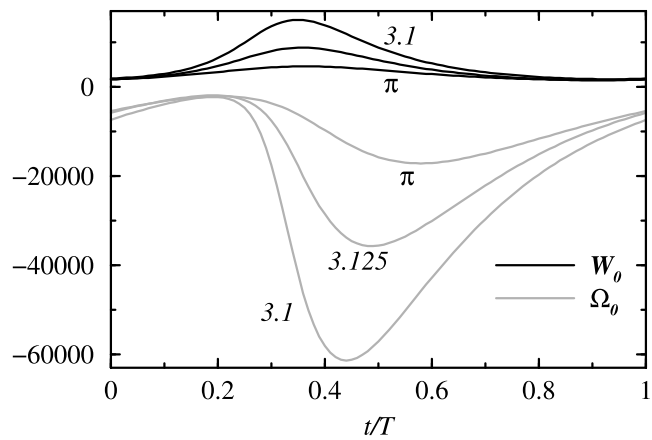

FIG. 11. (a) Hopf bifurcations (black disks) at $\alpha=\pi$ of $B_{1}, S_{1 p}$, and $S_{1 n}$, and continuation curves of the corresponding limit cycles $\Gamma, \Gamma_{p}$, and $\Gamma_{n} ;\langle V\rangle$ is the mean azimuthal speed $T^{-1} \int_{0}^{T} V(t) d t$. (b) Unfolding of the Hopf bifurcations of $S_{1 p}\left(H_{1 p}\right.$ curve), $S_{1 n}\left(H_{1 n}\right.$ curve), and $B_{1}\left(H_{1}\right.$ curve) around $\alpha=\pi$; black disks are codimension-two bifurcation points. The blue curves are the unfolding of the cyclic folds $C F$ in (a). (c) Time evolution of $W_{0}$ and $\Omega_{0}$ (axial and angular speed at the axis) for the periodic solutions $\Gamma$ and $\Gamma_{p}$ at $R=700$ and $\alpha=\pi$, shown as gray disks in (a). (d) Time evolution of $W_{0}$ and $\Omega_{0}$ for the periodic solution $\Gamma_{n}$ at $R=450$ and $\alpha$ values as indicated, shown as gray disks in (b).

moving away from the symmetry line $\alpha=\pi$. Bifurcation curves $H_{1 p}$ and $H_{1 n}$ cannot be continued beyond the limit points in Fig. 11(b) for the same reasons discussed before: both the unstable steady solutions $\left(S_{1 p}, S_{1 n}\right)$ and the bifurcated unstable limit cycles $\left(\Gamma_{p}, \Gamma_{n}\right)$ develop narrow inner shear layers at the axis, with large axial and angular velocities. The increase of the strength of the swirling axial jet is clearly seen in Fig. 11(d), showing the time evolution of $W_{0}$ and $\Omega_{0}$ for the periodic solution $\Gamma_{n}$ at $R=450$ and $\alpha=\pi, 3.125$, and 3.1, with periods $T_{\alpha=\pi}=5.242 \times 10^{-3}$, $T_{\alpha=3.125}=5.492 \times 10^{-3}$, and $T_{\alpha=3.1}=5.258 \times 10^{-3}$. This results in a lack of convergence of the Newton-Krylov-Poincaré continuation methods used, along with a deterioration of the accuracy of the corresponding solutions, requiring a higher number of Legendre polynomials. The strength of the oscillating axial jet at its maximum amplitude (see movie5.mp4 in the Supplemental Material [24]) resembles the $S_{3}$ steady solutions depicted in Fig. 7. These results strongly suggest that the swirling solutions $S_{1}$ and the corresponding bifurcated periodic orbits also have a divergence region, similar to the ones described in Fig. 20, but at higher Reynolds numbers, $R \gtrsim 400$.

Figure 12 is a close-up of Fig. 11(b) in the vicinity of $\alpha=\pi$. The bifurcation curves separate the parameter space in regions with different dynamics. These dynamics are illustrated in the phase 


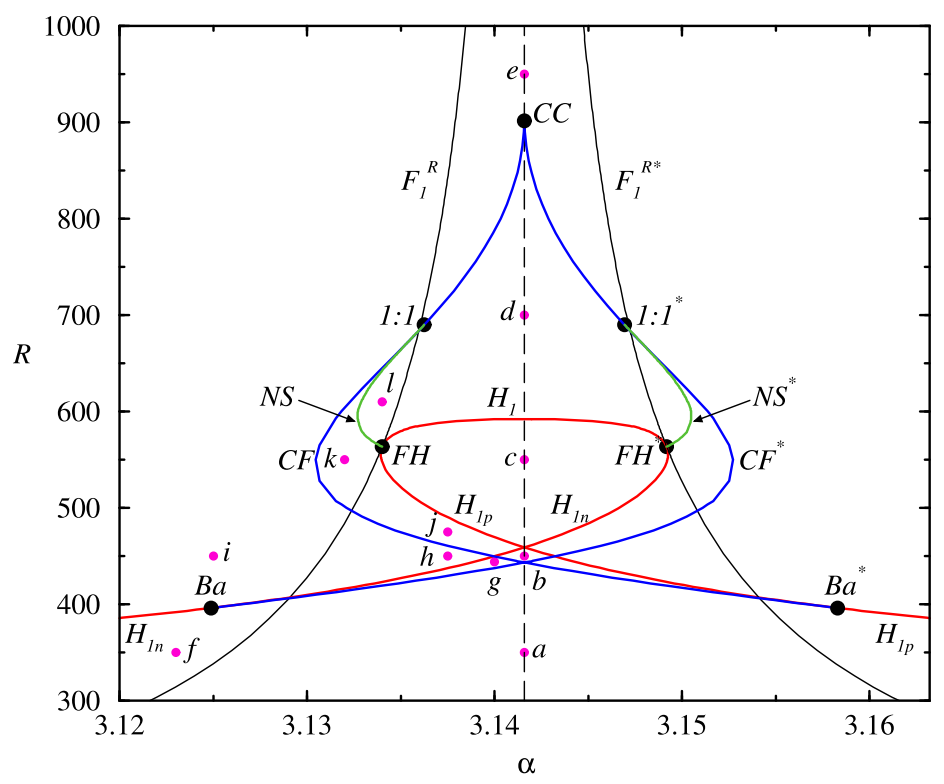

FIG. 12. Bifurcation diagram around $\alpha=\pi$. The magenta disks correspond to the phase portraits displayed in Figs. 13 and 14. Black disks are codimension-two cusp points.

portraits in Figs. 13 and 14, at the points indicated by magenta disks in each region. The phase portrait in Fig. 13(a), for $(\alpha, R)=(\pi, 350)$, shows the fixed points $B_{j}$ and $S_{j}, j=1-2$, that exist just before the Hopf bifurcations take place. $B_{1}$ and $S_{1}$ have positive velocity $W_{0}$ on the axis, while $B_{2}$ and $S_{2}$ have $W_{0}<0$. Initial conditions with $W_{0}=0$ evolve towards $W_{0}>0$, and therefore the dynamics for $W_{0}>0$ never interacts with the solutions on the region $W_{0}<0$. For this reason, we have only plotted phase portraits (from $b$ to $\ell$ ) involving the fixed points $B_{1}$ and $S_{1}$, and the limit cycles associated with them. In Fig. 13(a), $B_{1}$ is the only stable point, and we have plotted in black the stable manifold of $B_{1}$ that coincides with the unstable manifold of $S_{1 p}$ and $S_{1 n}$; arrows indicate time evolution. We have projected the trajectories on the plane $W_{0}=3000$ and the fixed points $B_{i}$ on the vertical plane $U=-25$ for clarity. According to Fig. 20(a), the solutions $S_{3}$ cannot be computed or do not exist in the parameter region explored in Fig. 12.

The first five phase portraits in Fig. 13 illustrate the limit cycle transitions when moving on the symmetry line $\alpha=\pi$ from $a$ to $e$ in Fig. 12. They correspond to five $R$-constant sections in Fig. 11(a) covering the five different regions in that diagram. All phase portraits are symmetric about the vertical plane $V=0$; the perspective may obscure this fact, but the projections on the coordinate planes make it clear enough.

The next four phase portraits, Fig. 13(f) and Figs. 14(g)-14(i), show the changes of the limit cycles when we move around the Bautin codimension-two point $B a$ [20], where the Hopf bifurcation $H_{1 n}$ changes from supercritical (to the left of $B a$ ) to subcritical (to the right of $B a$ ). From $f$ to $g$, we first cross the fold curve $F_{1}^{R}$ where $B_{1}$ and $S_{p}$ are born, and then the cyclic fold $C F^{*}$ where $\Gamma_{n}$ and $\Gamma_{\ell n}$ appear. From $g$ to $h, \Gamma_{\ell n}$ disappears when crossing $H_{1 n}$. From $h$ to $i, B_{1}$ and $S_{p}$ merge and disappear when crossing $C F^{*}$. There is an additional very narrow region in Fig. 12 to the right of $B a$, bounded by curves $C F^{*}, F_{1}^{R}$, and $H_{1 n}$, whose phase portrait is identical to $g$, except that $B_{1}$ and $S_{1 p}$ are absent.

The last three phase portraits in Fig. 14 describe the limit cycle transitions in the region between the cyclic fold curve $C F$ and the Hopf curves $H_{1 p}$ and $H_{1}$, for $\alpha<\pi$. From $b$ to $j, \Gamma_{\ell n}$ disappears when crossing $H_{1 n}$. From $j$ to $k, B_{1}$ and $S_{p}$ merge and disappear when crossing $C F^{*}$. From $k$ to $l$, the unstable limit cycle $\Gamma$ becomes stable when crossing the Neimark-Sacker bifurcation curve NS, to be discussed in the next section. Finally, from $l$ to $d, B_{1}$ and $S_{1 p}$ are born when crossing $F_{1}^{R}$. 
(a)

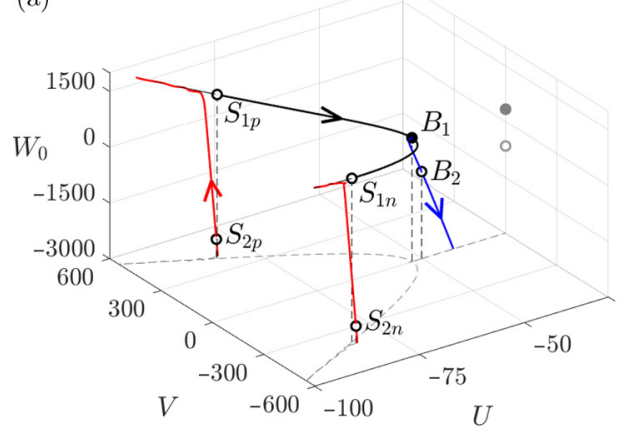

(c)

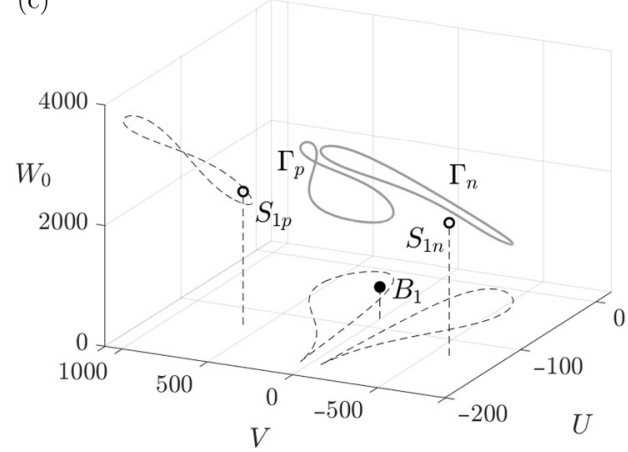

(e)

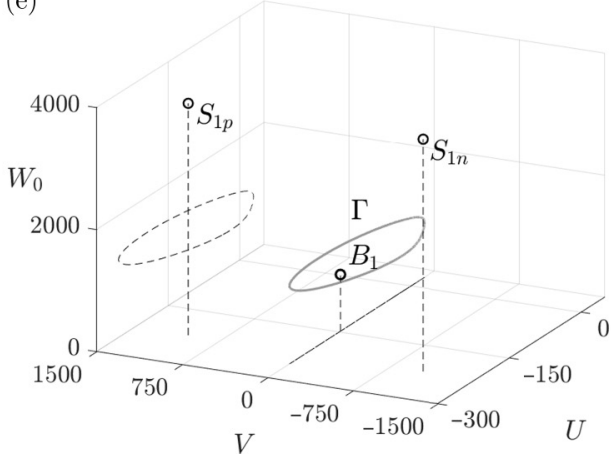

(b)

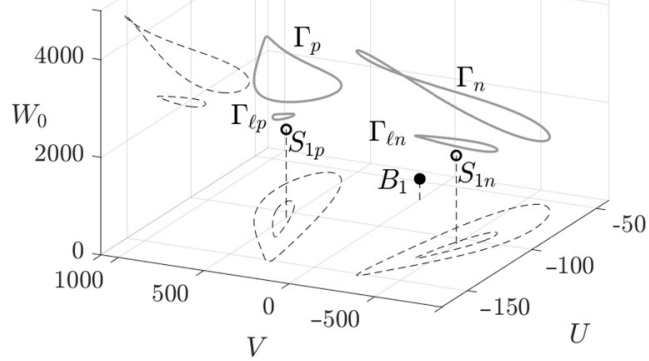

(d)

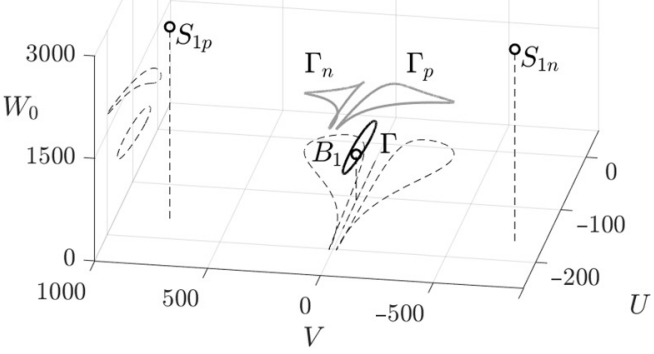

(f)

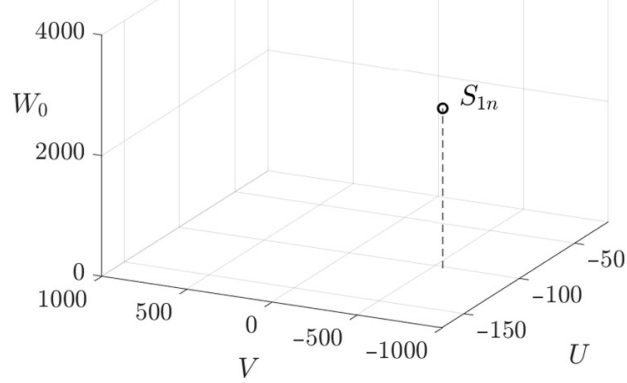

FIG. 13. Phase portraits, including fixed points and limit cycles, corresponding to the magenta disks $a-$ $f$ in the different regions of Fig. 12. Solid (hollow) symbols correspond to stable (unstable) fixed points; black (gray) curves indicate stable (unstable) limit cycles. The $(\alpha, R)$ values are (a) $(\pi, 350)$, (b) $(\pi, 450)$, (c) $(\pi, 550),(d)(\pi, 700),(e)(\pi, 950)$, and (f) $(3.123,350)$.

\section{QUASIPERIODIC SOLUTIONS}

In this section, we analyze the mechanism responsible for the stabilization of the limit cycle $\Gamma$ when moving from the phase portrait $k$ to $l$. We have computed the $N S$ boundary in Fig. 12 by monitoring the Floquet exponents (the eigenvalues of the limit cycle) of $\Gamma$ along continuation curves, using the Newton-Poincaré-Arnoldi method. We have found that a pair of complex-conjugate eigenvalues cross the unit circle, indicating that a Neimark-Sacker bifurcation takes place. In this 

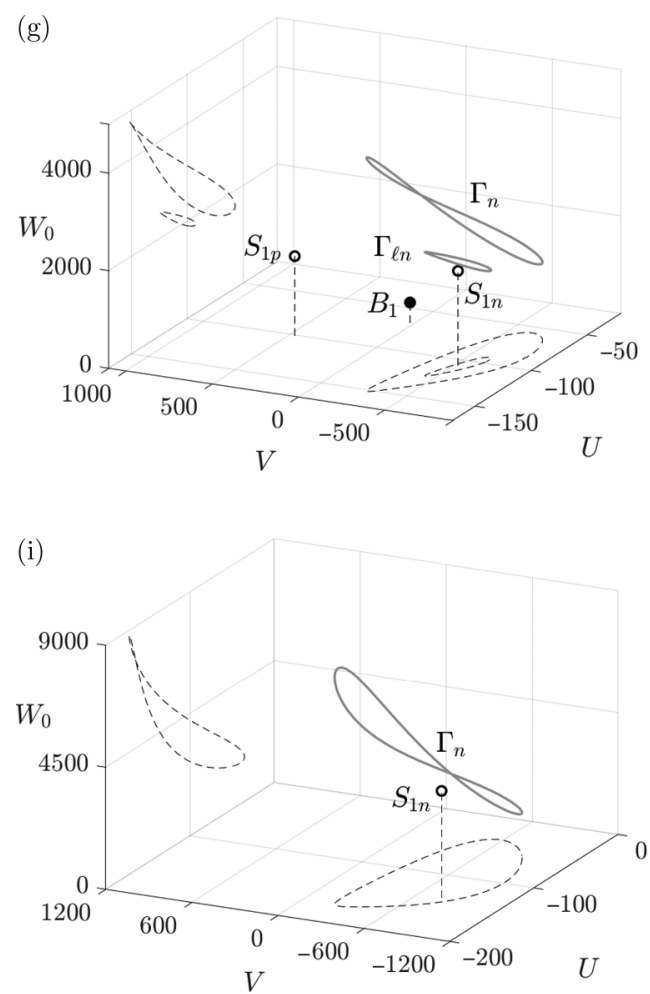

$(\mathrm{k})$

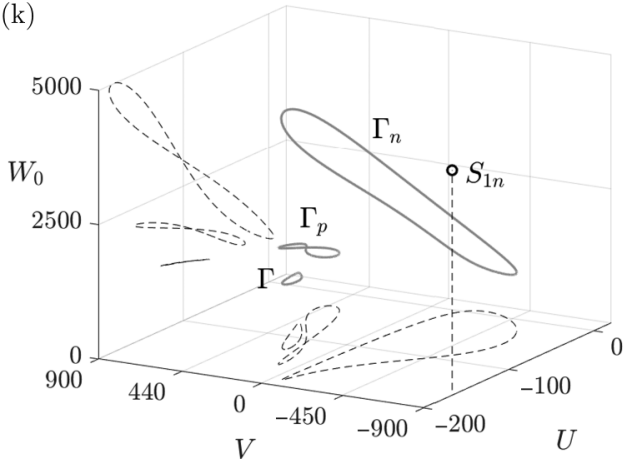

(h)

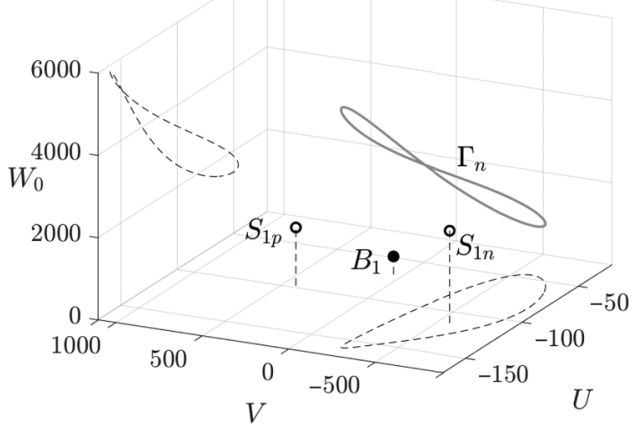

(j)

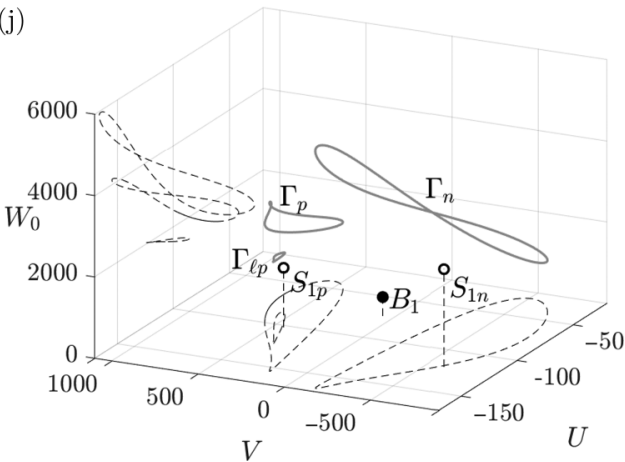

(1)

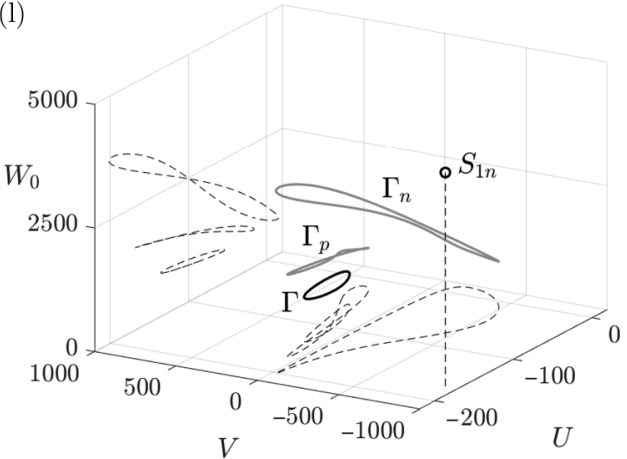

FIG. 14. Phase portraits, including fixed points and limit cycles, corresponding to the magenta disks $g-l$ in the different regions of Fig. 12. Solid (hollow) symbols correspond to stable (unstable) fixed points; black (gray) curves indicate stable (unstable) limit cycles. The $(\alpha, R)$ values are $(\mathrm{g})(3.14,444),(\mathrm{h})(3.1375,450)$, (i) $(3.125,450)$, (j) $(3.1375,475),(\mathrm{k})(3.132,550)$, and (1) $(3.134,610)$.

bifurcation, also called a Hopf bifurcation of limit cycles, a two-torus $T_{2}$ is born from the limit cycle, changing its stability.

If the quasiperiodic solution $T_{2}$ exists to the left (right) of curve $N S$ in Fig. 12, it should be stable (unstable), according to dynamical systems theory [20]. Our time integrations have not identified any stable quasiperiodic solution to the left of the boundary $N S$ and, therefore, the sought two-torus $T_{2}$ must necessarily be unstable and located to the right of that curve. In general, the computation of unstable tori arising in infinite-dimensional dynamical systems is a formidable task that requires sophisticated Newton-Poincaré algorithms. In this case, however, we can approach this torus to any 
(a)

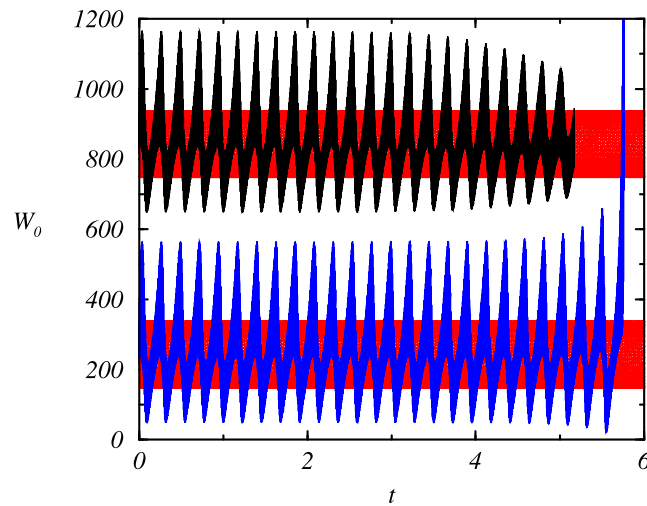

(c)

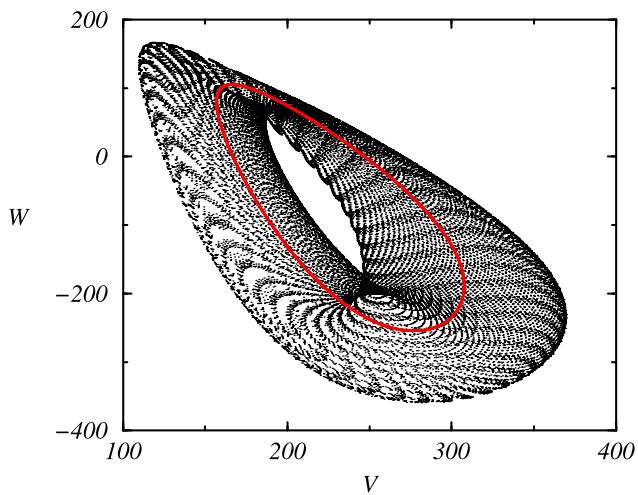

(b)

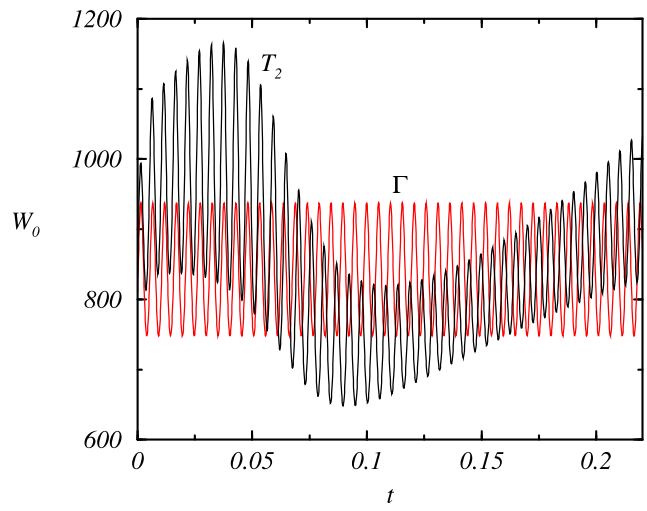

(d)

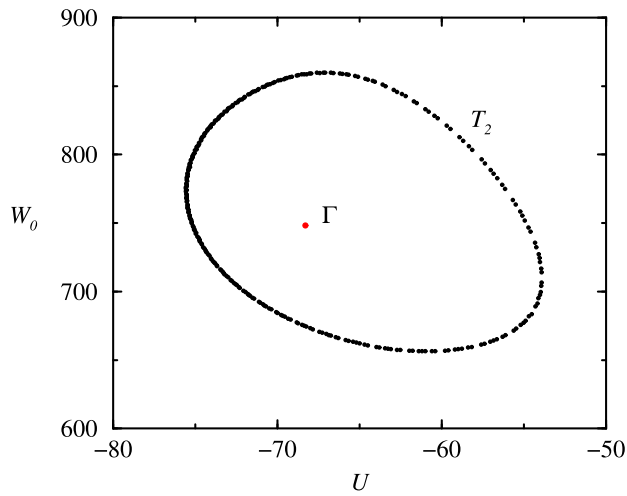

FIG. 15. Unstable two-torus $T_{2}$ born at the Neimark-Sacker bifurcation curve originating at the $F H$ point. (a) Time series starting very close to $T_{2}$; the black one evolves towards the stable limit cycle $\Gamma$ (shown in red), and the blue one escapes to infinity. The blue time series and the corresponding $T_{2}$ have been shifted -600 in $U$ for clarity. (b) Close-up of (a). (c) Phase portrait of $T_{2}$ and $\Gamma$ at $(\alpha, R)=(3.133,580)$, projected on the plane $(V, W)$. (d) Poincaré section of $T_{2}$ and $\Gamma$ in (c), at $W=-100$, projected on the plane $\left(U, W_{0}\right)$.

desired accuracy using the edge-state tracking [21,22]. The reason is that the linearly stable cycle $\Gamma$ has a surrounding basin of attraction of finite size whose boundary is partially constituted by the sought unstable two-torus $T_{2}$. As a consequence, any time integration initiated from a given point of $\Gamma$, accurately scaled by a factor $C \approx 1$, will either be attracted to $\Gamma$ or repelled outwards. Figure 15(a) shows two time evolutions: the black one for $C=1.0918890$, which falls back to $\Gamma$ (the red solution), and the blue one for $C=1.0918891$, which escapes to infinity. These time evolutions approach $T_{2}$ along its stable manifold, transiently spending very long lapses of time on this invariant set. This allows one to genuinely represent a phase portrait of the two-torus, shown in Fig. 15(c), along with its corresponding Poincaré section, shown in Fig. 15(d). The eigenvalues of $\Gamma$ in Fig. 15(c) closest to one are $\lambda_{ \pm}=0.984664 \pm 0.145889 i$, with modulus 0.995413 almost equal to one because we are very close to the NS curve. The imaginary part of $\lambda_{ \pm}$gives the second period and frequency of the torus, $\omega_{2}$ and $\tau_{2}$, in terms of the period and frequency of $\Gamma, \omega_{1}=1213.03$ and $\tau_{1}=0.00517972$ :

$$
\omega_{2}=\frac{\operatorname{Im} \lambda_{ \pm}}{2 \pi} \omega_{1}=28.1654, \quad \tau_{2}=0.223081 .
$$




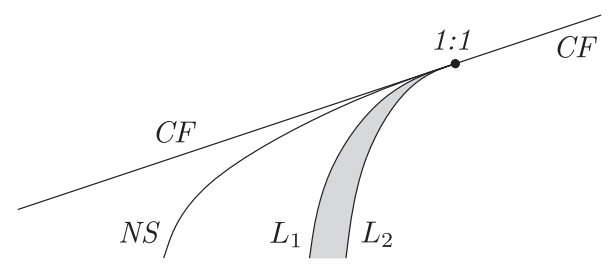

FIG. 16. Schematic of the $1: 1$ resonance codimension-two bifurcation. In the gray region between curves $L_{1}$ and $L_{1}$, a chaotic invariant set exists.

The new frequency $\omega_{2}$ is about 43 times smaller than $\omega_{1}$, which is clearly seen in the time series of Fig. 15(a): the fast oscillations associated with $\omega_{1}$ cannot be distinguished, and $\Gamma$ looks like a solid rectangle. Figure 15(b) is an enlarged part of Fig. 15(a), showing $T_{2}$ and $\Gamma$ over one period $\tau_{2}$.

The $N S$ curve is born at the fold-Hopf codimension-two point $F H$. The fold-Hopf bifurcation admits different scenarios [20]. In the present case, we have found that the limit cycle associated with the $F H$ bifurcation undergoes a Neimark-Sacker bifurcation, as indicated in Fig. 12. Therefore, we are in the fourth case discussed in [20], corresponding to Fig. 8.17. When increasing the Reynolds number $R$, the $N S$ curve becomes tangent to the fold curve $F_{1}^{R}$, and ends at the codimension-two bifurcation point $1: 1$ located at $(\alpha, R)=(3.1362,689.74)$; at this point, the imaginary part of the pair of complex-conjugate critical eigenvalues of $\Gamma$ is zero and we have a double-one eigenvalue, characteristic of the $1: 1$ resonance. This codimension-two bifurcation is the equivalent of the Takens-Bogdanov bifurcation of fixed points, but for limit cycles; see [20], Sec. 9.5.2, for details. The dynamical systems theory of the $1: 1$ resonance explains what the fate is of the $T_{2}$ quasiperiodic solution: it disappears in a homoclinic collision with the unstable limit cycle $\Gamma_{p}$, one of the limit cycles that appears at the cyclic fold $C F$. In fact, what is a homoclinic collision in the Takens-Bogdanov bifurcation of fixed points becomes a wedge of complexity for the 1:1 bifurcation for maps, as shown in the schematics of Fig. 16. Inside the wedge (gray region in the figure), the stable and unstable manifolds of the Poincaré map of $\Gamma_{p}$ intersect transversely, generating a chaotic invariant set, which is unstable in our case. The wedge is delimited by curves $L_{1}$ and $L_{2}$, where the stable and unstable manifolds of $\Gamma_{p}$ become tangent. The torus $T_{2}$ exists between the curves $N S$ and $L_{1}$; it becomes a chaotic invariant set at $L_{1}$ and the chaotic invariant set disappears at $L_{2}$.

\section{DISCUSSION}

In this work, we have explored the self-similar dynamics of a fluid contained in an infinite elastic pipe that is being axially stretched and azimuthally twisted, simultaneously. The inclusion of torsional effects has helped not only to find families of steady solutions, but also to understand their interaction in the twist-free case. We have found that the interesting dynamics take place around the symmetry lines $\alpha=0$ (the purely stretching case already studied in [4]) and $\alpha=\pi$, the purely shrinking case considered here. In these regions, up to eight different steady solutions exist and, around $\alpha=\pi$, complex temporal dynamics appear. The steady solutions obtained are all unstable in the self-similar subspace, except one of them $\left(B_{1}\right)$ for moderate $R$ values, or near $\alpha=\pi$. Away from the symmetry lines, the number of steady solutions decreases substantially via fold bifurcations, and the only additional bifurcation that takes place is a Hopf bifurcation resulting in an unstable limit cycle.

For moderate Reynolds number values $(R \leqslant 10)$, the eight solutions exist, with one of them stable, the solution smoothly connecting with the trivial solution at $R=0$. The existence of multiplicity of solutions at $R=0$, i.e., when the pipe wall is at rest, looks puzzling, but it is due to the unboundedness of the pipe (infinitely long) and of the velocity field, which grows linearly away from $z=0$ (self-similar formulation). If we make the domain finite, then additional boundary conditions 
(a)

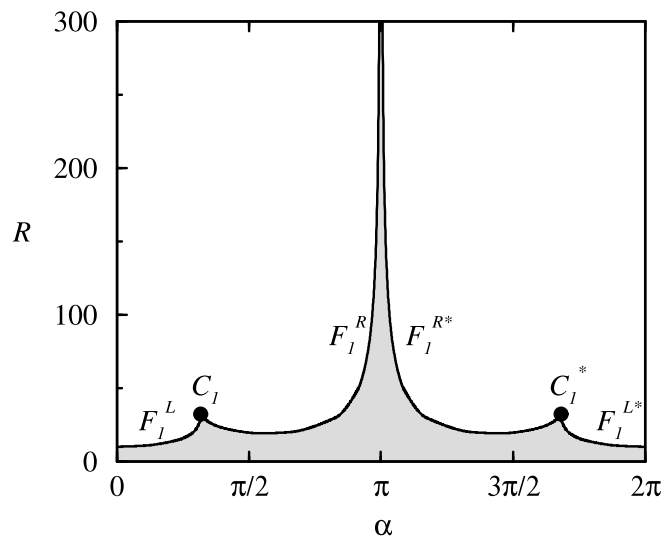

(b)

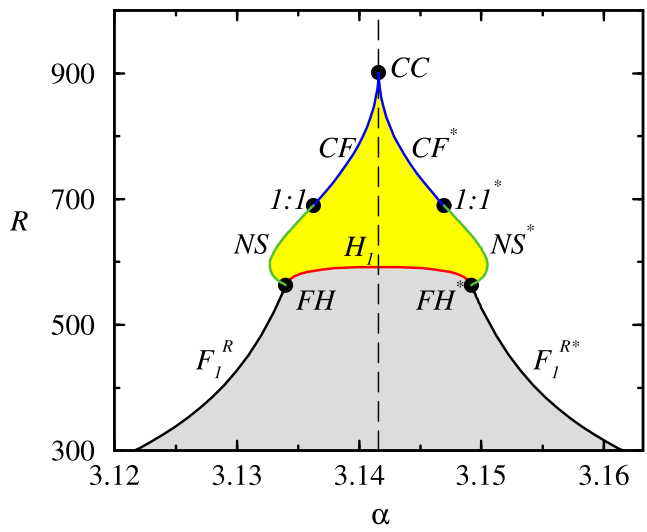

FIG. 17. Stable solutions. In gray, stability domain of the steady solution $B_{1}$; in yellow, stability domain of the periodic orbit $\Gamma$. (a) Parameter domain up to $R=300$. (b) Close-up around $\alpha=\pi$.

must be imposed at the pipe ends. Espín and Papageorgiu [19] performed such simulations in a channel flow, using the velocity field of a self-similar solution as boundary condition at the end walls. By doing that, energy and momentum are injected through the end walls and, by imposing different self-similar profiles, a multiplicity of different solutions can be obtained, even for $R=0$.

Former studies of extensional flows [4] in cylindrical geometry were mainly focused on the identification of steady flows (some of them including azimuthal swirl) for purely axial stretching only. In the present study, we have also explored the presence of time-periodic (limit cycles) and quasiperiodic flows (tori). Stable time-periodic flows have only been identified when the pipe is axially shrinking and with zero or very small torsion. This result is consistent with former DNS and self-similar computations in extensional planar channel flows that have identified these type of flows when the upper and lower plates are shrinking at the same deceleration rate $[11,19]$. Unstable quasiperiodic flows have also been computed by means of edge-state tracking techniques [21], suitably combined with Arnoldi linear stability analyses capable of shooting in the unstable eigen-Floquet mode.

Another salient feature of many of the flows obtained in this study is the presence of intense and narrow swirling axial jets, more pronounced in parameter regions with shrinking and twisting walls. The presence of this inner shear boundary layer results in serious computational problems, which make the continuation of these solutions very difficult, as shown in Fig. 20. These solutions are highly unstable even in the self-similar subspace, so they are going to be even more unstable against general perturbations, and unlikely to be observed in real flows.

We have only found two solutions stable in the self-similar subspace: the steady solution $B_{1}$, which we call the base state, and the periodic solution $\Gamma$. Figure 17 shows the parameter regions where these solutions are stable. The base state becomes unstable for small Reynolds numbers, $R \lesssim 25$, and, in fact, disappears in fold bifurcations $F_{1}$ [Fig. 17(a)], except near the $\alpha=\pi$ line, where it is stable up to $R \lesssim 570$ [Fig. 17(b)]. Above the fold bifurcation curves $F_{1}$ and away from the symmetry lines $\alpha=0$ and $\pi$, the number of solutions decreases when increasing $R$, and only a few steady solutions remain with no further bifurcations; the flow is very sensitive to symmetry breaking. Along the line $\alpha=0$, i.e., the purely stretching case, the number of steady solutions increases and are all unstable; we recover the previous results from [4], with an additional swirling branch $S_{3}$.

The dynamics become more complex around $\alpha=\pi$ when the pipe is mainly shrinking. The base state $B_{1}$ becomes unstable at the Hopf bifurcation $H_{1}$, where a stable limit cycle $\Gamma$ is born. A transitional region including complex dynamics takes place in the range $R \in[560,900]$. The limit 
cycle $\Gamma$ is stable in the yellow region shown in Fig. 17(b) and becomes unstable via two different instabilities, Neimark-Sacker bifurcations $N S$ and $N S^{*}$, and cyclic fold bifurcations, $C F$ and $C F^{*}$. These bifurcation curves meet at five codimension-two bifurcation points, indicated by black disks in Fig. 17(b), which have been described in Secs. V and VI. Complex dynamics including unstable two-torus and chaotic invariant sets have been found in this region. For Reynolds numbers above the transitional region, only unstable fixed points and limit cycles remain.

Flow-induced oscillations of fluids in elastic-walled conduits arise in many industrial and biological systems, as we mentioned in Sec. I [7,8]. The stable periodic solutions that we have obtained for purely shrinking and slightly twisted pipes [the yellow region in Fig. 17(b)] are an example of such flow-induced oscillations. Therefore, we have identified a mechanism that may potentially generate pulsating time-periodic stable flows in a shrinking pipe. Since the region of existence of these oscillatory flows extends within a range of moderate Reynolds numbers, they could potentially be detected in direct numerical simulations (DNSs) of the full Navier-Stokes equations. We believe that these oscillations are associated with the formation of intense jets at the pipe axis. When the intensity of the jet is large, it becomes unstable and results in the observed stable oscillations.

Previous DNS computations carried out in channel flows [19] have reproduced some of the solutions found in the self-similar subspace. However, this work has also pointed out that when increasing $R$, the resulting DNS computed flows may differ from the self-similar bifurcated solutions. In cylindrical geometries, this comparison is still a pending and challenging task.

\section{ACKNOWLEDGMENTS}

This work was supported by the Spanish MINECO Grants No. FIS2017-85794-P and No. PRX18/00179, and the Generalitat de Catalunya Grant No. 2017-SGR-785.

\section{APPENDIX A: DETAILS OF THE SELF-SIMILAR FORMULATION}

The axisymmetric Navier-Stokes equations in cylindrical coordinates $(r, \theta, z)$ plus incompressibility are

$$
\begin{aligned}
u_{t}+u u_{r}+w u_{z}-r^{-1} v^{2} & =-p_{r}+d d_{+} u+u_{z z}, \\
v_{t}+u d_{+} v+w v_{z} & =d d_{+} v+v_{z z} \\
w_{t}+u w_{r}+w w_{z} & =-p_{z}+d_{+} d w \\
d_{+} u+w_{z} & =0
\end{aligned}
$$

where $d=\partial_{r}, d_{+}=\partial_{r}+1 / r=r^{-1} \partial_{r} r$. The incompressibility condition and the linearity of $w$ with $z$ (self-similar ansatz) results in $w_{z}$ and $u$ independent of $z$; therefore, $w=-z d_{+} u=-z r^{-1}=$ $-2 z D_{+} F$, where we have used $u=r F(x, t)$. Substituting the ansatz (2) into the Navier-Stokes equations (A1a)-(A1c), we obtain

$$
\begin{aligned}
& r F_{t}+r F d(r F)-r(z H)^{2}=-p_{r}+8 r D D_{+} F \\
& \quad \Rightarrow \quad p_{r}=r\left\{8 D D_{+} F-2 F D_{+} F+F^{2}+(z H)^{2}-F_{t}\right\} \\
& r(z H)_{t}+2 r F d_{+}(z H)-2 r H D_{+}(z F)=8 r D D_{+}(z H) \\
& \quad \Rightarrow \quad H_{t}+2 F D_{+} H-2 H D_{+} F=8 D D_{+} H, \\
& -2 D_{+}(z F)_{t}-4(x+1) F D D_{+}(z F)+4\left(D_{+} F\right) D_{+}(z F)=-p_{z}-16 D_{+} D D_{+}(z F) \\
& \quad \Rightarrow p_{z}=2 z\left\{D_{+} F_{t}+2(x+1) F D D_{+} F-2\left(D_{+} F\right)^{2}-8 D_{+} D D_{+} F\right\} .
\end{aligned}
$$

The integrability condition for $p$ from (A2b) and (A2e) results in an evolution equation for $F$,

$$
D D_{+} F_{t}+2 D_{+}\left(F D D_{+} F\right)-4\left(D_{+} F\right) D D_{+} F-\frac{1}{4} H^{2}=8\left(D D_{+}\right)^{2} F .
$$


We can compute the pressure, $p(r, \theta, z, t)=z^{2} p_{2}(x, t)+2 z p_{1}(x, t)+p_{0}(x, t)$, with $p_{2}$ and $p_{0}$ given by (6) and (7). The integration constant in the $p_{0}$ term is the gauge freedom of the pressure for incompressible flow.

For axisymmetric problems, as is the case for the self-similar formulation, there is a stream function $\psi$, given by

$$
\begin{aligned}
\partial_{z} \psi & =-r u=-\frac{1}{2}(x+1) F, \quad \partial_{r} \psi=4 r D \psi=r w=-2 r z D_{+} F \\
& =-2 r z D((x+1) F) \quad \Rightarrow \quad \psi(x, z, t)=-\frac{1}{2}(x+1) z F .
\end{aligned}
$$

The Hermitian product of two arbitrary integrable functions $\varphi(x)$ and $\psi(x)$ within the domain $x \in[-1,1]$ is defined as

$$
\langle\varphi, \psi\rangle=\int_{-1}^{1} \varphi(x) \psi(x) d x .
$$

The explicit matrices and vectors appearing in the dynamical system of amplitudes (17) are

$$
\begin{gathered}
A_{m n}=\left\langle\Phi_{m}, D D_{+} \Phi_{n}\right\rangle, \quad G_{2, m n}=\left\langle\Psi_{m}, \Psi_{n}\right\rangle, \\
L_{1, m n}=\left\langle\Phi_{m}, 8\left(D D_{+}\right)^{2} \Phi_{n}\right\rangle, \quad L_{2, m n}=\left\langle\Psi_{m}, 8 D D_{+} \Psi_{n}\right\rangle, \\
N_{1, m}=\left\langle\Phi_{m}, N_{f}\right\rangle, \quad N_{2, m}=\left\langle\Psi_{m}, N_{h}\right\rangle .
\end{gathered}
$$

These inner products are computed to spectral accuracy using Gauss-Legendre quadrature formulas [23].

\section{APPENDIX B: CONVERGENCE ISSUES}

The selection of the number of polynomials used in the spectral expansions (16) has been a critical issue in the continuation process. We have monitored the convergence of the Newton method by requiring a relative residual norm of $10^{-9}$ (ratio between the $L_{2}$ norm of the residual and the $L_{2}$ norm of the solution obtained). However, this has not proved enough to ensure a satisfactory spectral convergence. We measure the spectral convergence as the gap between the maximum and minimum spectral coefficients, for modes $a_{m}$ and $c_{m}$. For example, Fig. 18(a) shows the amplitudes of the spectral coefficients of an exponentially converged solution. It is the solution of the $S_{1 n}$ branch at $R=120, \alpha=\pi / 2$, and $M=70$ polynomials, with a spectral gap of about $10^{8}$; that is, the amplitudes of the modes $a_{m}$ and $c_{m}$ decay at least eight orders of magnitude. By contrast, Fig. 18(b) shows the amplitudes for another solution of the same branch, the same value of $M$, and the same Reynolds number $R$, but for a different value of $\alpha$, namely, $\alpha=3 \pi / 4$. This solution has a very poor spectral convergence of only two orders of magnitude, and clearly the decay is not exponential. In order to improve the accuracy of this solution, we have increased the number of polynomials. For $M=140$ [Fig. 18(c)], the spectral gap is of about $10^{4}$, whereas for $M=200$ [Fig. 18(d)], the spectral gap increases to $10^{7}$. All computations reported in the present paper are considered to be spectrally converged if their spectral gap is at least of four orders of magnitude.

The physical reason for the striking difference in spectral convergence between Fig. 18(a) at $\alpha=\pi / 2$ and Figs. 18(b)-18(d) at $\alpha=3 \pi / 4$ is that in this last case, the flow develops an intense and narrow inner shear layer at the cylinder axis, with large axial and angular velocity, which are one order of magnitude larger than in the $\alpha=\pi / 2$ case, as shown in Fig. 19. In addition, the axial jet is narrower for $\alpha=3 \pi / 4$, with a diameter about half of the diameter in the $\alpha=\pi / 2$ case.

By default, the solutions presented in this paper have all been computed with at least $M=70$ Legendre polynomials. During the continuation process of these solutions, the spectral gap has been monitored and the number of polynomials accordingly increased to $M=140$ or $M=200$ whenever the threshold limit of $10^{4}$ spectral gap decay has been reached. For $M>200$, the Legendre 
(a)

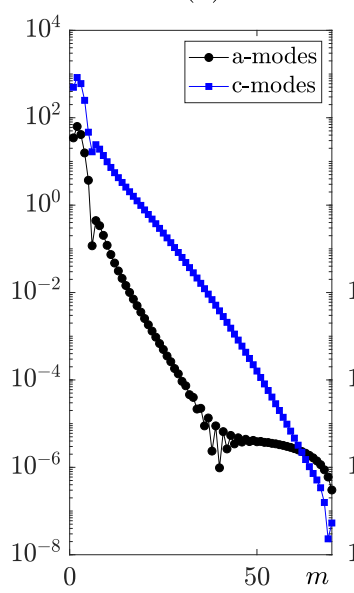

(b)

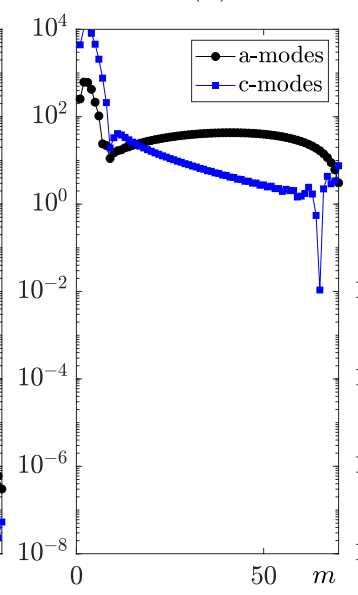

(c)

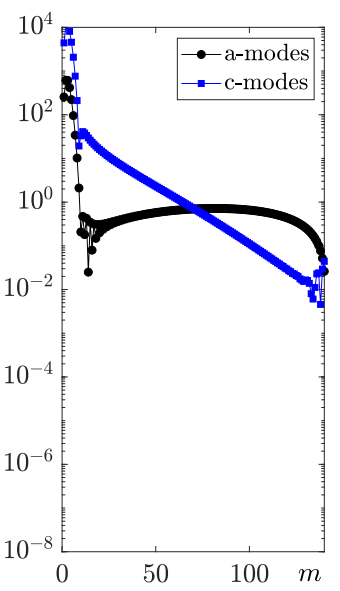

(d)

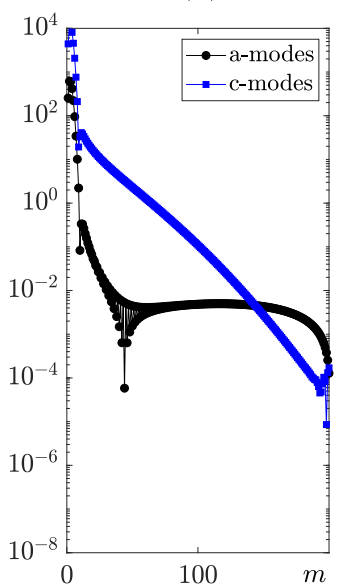

FIG. 18. Spectral convergence. Amplitudes of the spectral coefficients $a_{m}$ and $c_{m}$ in the expansions (16), for $S_{1 n}$ at $R=120$, and $\alpha$ and $M$ as indicated. (a) $\alpha=\pi / 2, M=70$, (b) $\alpha=3 \pi / 4, M=70$, (c) $\alpha=3 \pi / 4, M$ $=140$, and $(\mathrm{d}) \alpha=3 \pi / 4, M=200$.

differentiation matrices become extremely ill conditioned and the spectral gap does not improve, overall deteriorating the accuracy of the solutions.

When increasing the Reynolds number, some solutions belonging to the $S_{2}$ and $S_{3}$ branches exhibit a deterioration in the spectral gap when approaching the region between $\pi / 2$ and $3 \pi / 2$. In that region, it has been impossible to satisfy the four orders of magnitude of spectral decay criterion, even with $M=200$ polynomials. This deterioration is also due to the formation and growth of intense narrow shear layers at $r=0$. Figures 20(a) and 20(b) describe the growth of the axial and angular velocities at the pipe axis, $W_{0}$ and $\Omega_{0}$, for solutions $S_{3 p}$ and $S_{2 n}$ approaching the threshold criterion for spectral convergence. Figure 20(a) shows that these growth rates are superexponential for $S_{3 p}$ at $R=120$ and increasing $\alpha$; the fitted curves in red are of the form $a /\left(\alpha_{d i v}-\alpha\right)^{b}$, suggesting that the $S_{3 p}$ solutions escape to infinity for $\alpha \rightarrow \alpha_{d i v}=1.364$, and these solutions cease to exist beyond this critical value. The growth rate of $S_{2 n}$ shown in Fig. 20(b) is not so abrupt, but it is still exponential, as the fitted curves in red show. The end points of the continuation curves in Figs. 20(a) and 20(b) indicate the $\alpha$ values beyond which the spectral convergence criterion cannot be satisfied.

This convergence criterion has been systematically applied to all the solution branches explored in this paper. As a result, some of the solutions shown in Fig. 2 cannot be continued over the whole range $\alpha=[0,2 \pi]$. For $R \geqslant 30$, some of the continuation curves stop at aiven $\alpha$ value without any bifurcation taking place, precisely where the convergence criterion fails. Figure 20(c) shows the curves in the $(\alpha, R)$ where the convergence criterion is at stake (orange and brown solid and
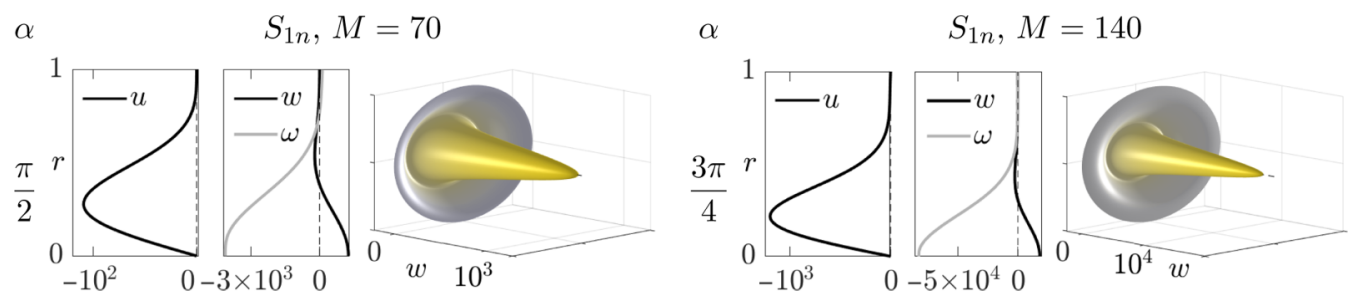

FIG. 19. Structure of the steady solutions $S_{1 n}$ at $R=120$, for the $\alpha$ and $M$ values indicated. The plots follow the same conventions as in Fig. 4. 
(a)

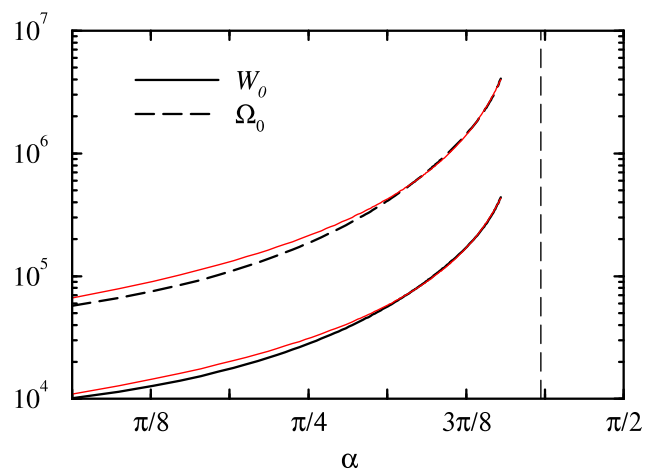

(b)

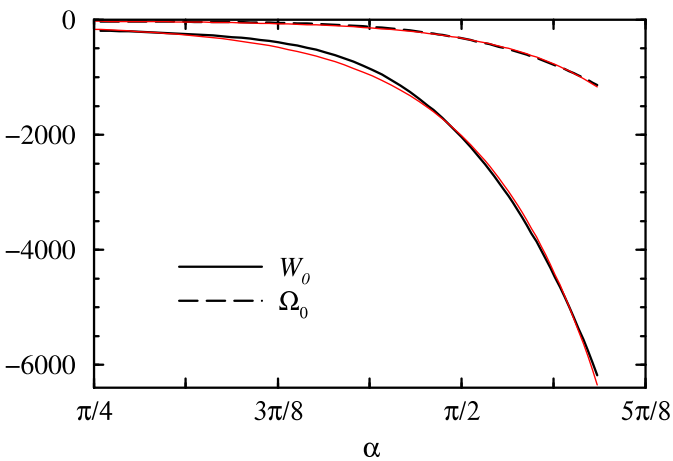

(c)

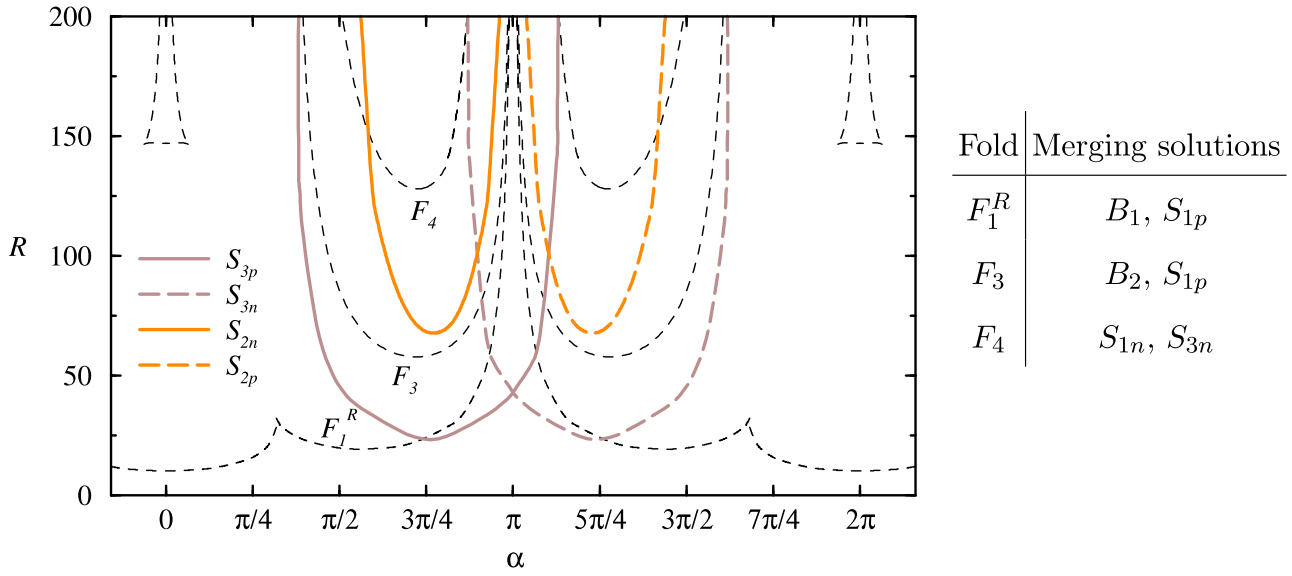

FIG. 20. Variation of $W_{0}$ and $\Omega_{0}$ with $\alpha$ approaching the divergence boundaries for (a) $S_{3 p}$ and (b) $S_{2 n}$, at $R=120$. The fits shown in red are (a) $W_{0}=8.575 \times 10^{4}(1.364-\alpha)^{-1.495}, \Omega_{0}=1.373 \times$ $10^{4}(1.364-\alpha)^{-1.665}$ and (b) $W_{0}=-19.31-0.2188 \exp (4.605 \alpha), \Omega_{0}=-87.83-3.267 \exp (4.063 \alpha)$. (c) Divergence boundaries of the $S_{3 p}, S_{3 n}, S_{2 p}$, and $S_{2 n}$ branches. The dashed black lines are the bifurcation curves in Fig. 8 .

dashed curves). Inside the region bounded by these curves, the solutions indicated in the figure legend cannot be spectrally converged to the required accuracy.

Although different convergence requirements would lead to different boundaries, Figs. 20(a) and 20(b) clearly indicate that inside these regions, the axial and angular velocities at the pipe axis growth exponentially or even superexponentially. This is a clear indication of the existence of a genuine physical divergence of the self-similar solutions. Nevertheless, none of the fold bifurcations occurring inside the divergence regions and shown in Fig. 20(c) as dashed black lines involve these pathological solutions (they belong to other solution branches, as indicated in the table of Fig. 20), so it has been possible to compute them even inside these regions.

Overall, branches $B_{1}$ and $B_{2}$ were computed with $M=70$ Legendre polynomials. Branch $S_{1}$ was required to increase this number up to $M=140$ in some $(\alpha, R)$ regions. Finally, the number of modes required for branches $S_{2}$ and $S_{3}$ ranged within the interval $M \in[70,200]$ and exhibit a divergence region. The convergence difficulties are associated with the torsion of the pipe and the conflicting regions are located in the region $\alpha \in(\pi / 2,3 \pi / 2)$, as shown in Fig. 20(c). However, the torsion-free case $\alpha=0$, studied previously, shows much better convergence. We have recovered 
all branches found in [4] using $M=70$, and for the branch $S_{3}, M=140$ was enough, even for $R=3000$ (see Fig. 3).

[1] S. L. Waters, Solute uptake through the walls of a pulsating channel, J. Fluid Mech. 433, 193 (2001).

[2] S. L. Waters, A mathematical model for the laser treatment of heart disease, J. Biomech. 37, 281 (2004).

[3] P. G. Drazin and N. Riley, The Navier-Stokes Equations, A Classification of Flows and Exacts Solutions (Cambridge University Press, Cambridge, 2006).

[4] J. F. Brady and A. Acrivos, Steady flow in a channel or tube with an accelerating surface velocity. An exact solution to the Navier-Stokes equations with reverse flow, J. Fluid Mech. 112, 127 (1981).

[5] M. B. Zaturska and W. H. H. Banks, Flow in a pipe driven by suction at an accelerating wall, Acta Mech. 110, 111 (1995).

[6] W. H. H. Banks and M. B. Zaturska, Swirling flow in a porous pipe with an accelerating wall, Acta Mech. 119, 1 (1996).

[7] P. S. Stewart, S. L. Waters, and O. E. Jensen, Local and global instabilities of flow in a flexible-walled channel, Eur. J. Mech. - B Fluids 28, 541 (2009).

[8] R. J. Whittaker, M. Heil, O. E. Jensen, and S. L. Waters, Predicting the onset of high-frequency selfexcited oscillations in elastic-walled tubes, Proc. R. Soc. London A 466, 3635 (2010).

[9] E. B. B. Watson, W. H. H. Banks, M. B. Zaturska, and P. G. Drazin, On transition to chaos in twodimensional channel flow symmetrically driven by accelerating walls, J. Fluid Mech. 212, 451 (1990).

[10] P. Hall and D. T. Papageorgiou, The onset of chaos in a class of Navier-Stokes solutions, J. Fluid Mech. 393, 59 (1999).

[11] F. Marques, A. Meseguer, F. Mellibovsky, and P. Weidman, Extensional channel flow revisited: A dynamical systems perspective, Proc. R. Soc. London A 473, 20170151 (2017).

[12] A. Leonard and A. Wray, in Proceedings of the Eighth International Conference on Numerical Methods in Fluid Dynamics, edited by E. Krause (Springer-Verlag, Berlin, 1982), p. 335.

[13] V. G. Priymak and T. Miyazaki, Accurate Navier-Stokes investigation of transitional and turbulent flows in a circular pipe, J. Comput. Phys. 142, 370 (1998).

[14] A. Meseguer and L. N. Trefethen, Linearized pipe flow to Reynolds number 10 ${ }^{7}$, J. Comput. Phys. 186, 178 (2003).

[15] T. Matsushima and P. Marcus, A spectral method for polar coordinates, J. Comput. Phys. 120, 365 (1995).

[16] C. Kelley, Solving Nonlinear Equations with Newton's Method (SIAM, Philadelphia, 2003).

[17] F. Mellibovsky and A. Meseguer, A mechanism for streamwise localisation of nonlinear waves in shear flows, J. Fluid Mech. 779, R1 (2015).

[18] L. N. Trefethen and D. Bau, Numerical Linear Algebra, (SIAM, Philadelphia, 1997).

[19] L. Espín and D. T. Papageorgiou, Flow in a channel with accelerating or decelerating wall velocity: A comparison between self-similar solutions and Navier-Stokes computations in finite domains, Phys. Fluids 21, 113601 (2009).

[20] Y. A. Kuznetsov, Elements of Applied Bifurcation Theory, 3rd ed. (Springer, New York, 2004).

[21] J. D. Skufca, J. A. Yorke, and B. Eckhardt, Edge of Chaos in a Parallel Shear Flow, Phys. Rev. Lett. 96, 174101 (2006).

[22] F. Mellibovsky, A. Meseguer, T. M. Schneider, and B. Eckhardt, Transition in Localized Pipe Flow Turbulence, Phys. Rev. Lett. 103, 054502 (2009).

[23] G. H. Golub and J. H. Welsch, Calculation of Gauss quadrature rules, Math. Comput. 23, 221 (1969).

[24] See Supplemental Material at http://link.aps.org/supplemental/10.1103/PhysRevFluids.4.103908 for the movies animating some of the branches of steady and periodic solutions found (movie1.mp4 to movie5.mp4). 